\title{
THE ACS SURVEY OF GALACTIC GLOBULAR CLUSTERS. XI. THE THREE-DIMENSIONAL ORIENTATION OF THE SAGITTARIUS DWARF SPHEROIDAL GALAXY AND ITS GLOBULAR CLUSTERS
}

\author{
Michael H. Siegel ${ }^{1}$, Stevenen R. Majewski ${ }^{2}$, David R. Law ${ }^{3,12}$, Ata Sarajedini ${ }^{4}$, Aaron Dotter ${ }^{5,6}$, A. Marín-Franch ${ }^{7}$, \\ Brian Chaboyer ${ }^{8}, \mathrm{JAy}_{\text {Anderson }}^{6}$, Antonio Aparicio ${ }^{7}$, Luigi R. Bedin ${ }^{9}$, Maren Hempel $^{10}$, Antonino Milone ${ }^{11}$, \\ Nathaniel Paust ${ }^{6}$, Giampaolo Piotto ${ }^{11}$, I. Neill Reid ${ }^{6}$, And Alfred Rosenberg ${ }^{7}$ \\ ${ }^{1}$ Department of Astronomy and Astrophysics, The Pennsylvania State University, 525 Davey Laboratory, University Park, PA 16802, USA; siegel@astro.psu.edu \\ ${ }^{2}$ Department of Astronomy, University of Virginia, P.O. Box 400325, Charlottesville, VA 22904-4325, USA; srm4n@ virginia.edu \\ ${ }^{3}$ Department of Physics and Astronomy, University of California, Los Angeles, CA 90095, USA; drlaw@astro.ucla.edu \\ ${ }^{4}$ Department of Astronomy, University of Florida, 211 Bryant Space Science Center, Gainesville, FL 32611, USA; ata@ astro.ufl.edu \\ ${ }^{5}$ Department of Physics and Astronomy, Elliott Building, 3800 Finnerty Road, Victoria, BC V8P 5C2, Canada; dotter@uvic.ca \\ ${ }^{6}$ Space Telescope Science Institute, 3700 San Martin Drive, Baltimore, MD 21218, USA; \\ dotter@stsci.edu, jayander@stsci.edu, bedin@stsci.edu,paust@stsci.edu,inr@stsci.edu \\ ${ }^{7}$ University of La Laguna and Instituto de Astrofísica de Canarias, Vía Láctea s/n, E-38200 La Laguna, Spain; amarin@iac.es, antapaj@iac.es, alf@iac.es \\ ${ }^{8}$ Department of Physics and Astronomy, Dartmouth College, 6127 Wilder Laboratory, Hanover, NH 03755, USA; chaboyer@ heather.dartmouth.edu \\ ${ }_{9}^{9}$ Astronomical Observatory of Padova, Vicolo dell'Osservatorio 5, I-35122 Padova, Italy; luigi.bedin@ oapd.inaf.it \\ ${ }^{10}$ Departamento de Astronomia y Astrofisica, Vic. Mackenna 4860 Casilla 306, Santiago, 22, Chile; mhempel@astro.puc.cl \\ ${ }^{11}$ Dipartimento di Astronomia, Università di Padova, I-35122 Padova, Italy; antonino.milone@oapd.inaf.it, giampaolo.piotto@ unipd.it \\ Received 2011 May 3; accepted 2011 August 25; published 2011 November 18
}

\begin{abstract}
We use observations from the Hubble Space Telescope Advanced Camera for Surveys (HST/ACS) study of Galactic globular clusters to investigate the spatial distribution of the inner regions of the disrupting Sagittarius dwarf spheroidal galaxy (Sgr). We combine previously published analyses of four Sgr member clusters located near or in the Sgr core (M54, Arp 2, Terzan 7, and Terzan 8) with a new analysis of diffuse Sgr material identified in the background of five low-latitude Galactic bulge clusters (NGC 6624, 6637, 6652, 6681, and 6809) observed as part of the ACS survey. By comparing the bulge cluster color-magnitude diagrams to our previous analysis of the M54/Sgr core, we estimate distances to these background features. The combined data from four Sgr member clusters and five Sgr background features provide nine independent measures of the Sgr distance and, as a group, provide uniformly measured and calibrated probes of different parts of the inner regions of Sgr spanning $20^{\circ}$ over the face of the disrupting dwarf. This allows us, for the first time, to constrain the three-dimensional orientation of Sgr's disrupting core and globular cluster system and compare that orientation to the predictions of an $N$-body model of tidal disruption. The density and distance of Sgr debris are consistent with models that favor a relatively high Sgr core mass and a slightly greater distance $(28-30 \mathrm{kpc}$, with a mean of $29.4 \mathrm{kpc})$. Our analysis also suggests that M54 is in the foreground of Sgr by $\sim 2 \mathrm{kpc}$, projected on the center of the Sgr dSph. While this would imply a remarkable alignment of the cluster and the Sgr nucleus along the line of sight, we cannot identify any systematic effect in our analysis that would falsely create the measured $2 \mathrm{kpc}$ separation. Finally, we find that the cluster Terzan 7 has the most discrepant distance $(25 \mathrm{kpc})$ among the four $\mathrm{Sgr}$ core clusters, which may suggest a different dynamical history than the other Sgr core clusters.
\end{abstract}

Key words: galaxies: individual (Sgr dSph) - galaxies: star clusters: general - Galaxy: evolution - globular clusters: general - globular clusters: individual (M54, Arp 2, Terzan 7, Terzan 8)

Online-only material: color figures

\section{INTRODUCTION}

The Sagittarius dwarf spheroidal galaxy (Sgr) is among the nearest known dwarf galaxies to the Milky Way. Soon after its discovery (Ibata et al. 1994), it became obvious that Sgr had an extended surface brightness profile consistent with the expected profile of a dwarf galaxy merging with the Milky Way. Its tidal tails have now been traced around the entire celestial sphere (see, e.g., Majewski et al. 2003, hereafter M03; Belokurov et al. 2006; Yanny et al. 2009 and references therein; Correnti et al. 2010) and it has become a paradigm of the hierarchical formation of the outer Milky Way.

Recent wide-field surveys and $N$-body merging simulations of Sgr have done an excellent job of measuring and constraining the overall placement and dynamics of the extended tidal tails of Sgr, with concomitant insight into the shape of the Milky

\footnotetext{
${ }^{12}$ Hubble Fellow.
}

Way's dark matter halo (see, e.g., Ibata et al. 2001; Helmi 2004; Law et al. 2005, 2009; Johnston et al. 2005; Fellhauer et al. 2006; Martínez-Delgado et al. 2007; Law \& Majewski 2010a, hereafter LM10a), the association of stellar structures within the halo (Bellazzini et al. 2003a, 2003b; Law et al. 2005; LM10a; Law \& Majewski 2010b, hereafter LM10b), and the status of potential Sgr debris within a few kiloparsecs of the Sun (Law et al. 2005; Yanny et al. 2009; LM10a). However, many questions remain unanswered regarding Sgr's structure and chemodynamical history. Sgr is the most elliptical of the dSphs and the origin of this ellipticity is unclear. What is the relative distribution of dark and luminous matter? Is the current Sgr core oblate or prolate? Does the postulated bifurcation of the stream result from substructure in the satellite, as suggested by LM10a, or from a disk structure in the initial satellite, as suggested by Peñarrubia et al. (2010; but cf. Łokas et al. 2010; Peñarrubia et al. 2011; P. Frinchaboy et al. 2011, in preparation)? Are the globular clusters associated with Sgr 
Table 1

Sagittarius Member Clusters

\begin{tabular}{lrccccccc}
\hline \hline Cluster & $\begin{array}{c}\Lambda_{\odot} \\
(\mathrm{deg})\end{array}$ & $\begin{array}{c}B_{\odot} \\
(\mathrm{deg})\end{array}$ & {$[\mathrm{Fe} / \mathrm{H}]$} & {$[\alpha / \mathrm{Fe}]$} & Age & $(m-M)_{0}$ & $E(B-V)$ & $\begin{array}{c}\text { Distance } \\
(\mathrm{kpc})\end{array}$ \\
\hline Arp 2 & 7.2 & 0.4 & -1.89 & +0.31 & 13.5 & 17.37 & 0.09 & $29.4 \pm 0.7$ \\
Terzan 7 & 5.4 & 4.9 & -0.60 & -0.03 & 8.0 & 17.05 & 0.06 & $25.3 \pm 0.6$ \\
Terzan 8 & 10.3 & 3.8 & -2.30 & +0.37 & 13.0 & 17.26 & 0.14 & $28.3 \pm 0.7$ \\
M54 & 0.0 & 1.5 & -1.80 & +0.20 & 13.5 & 17.27 & 0.14 & $28.4 \pm 0.7$ \\
\hline
\end{tabular}

(M54, Terzan 7, Terzan 8, Arp 2) bound or unbound to Sgr? Was the Sgr progenitor similar to the Small Magellanic Cloud or Large Magellanic Cloud, as suggested by its stellar chemistry (Chou et al. 2010) and current total luminosity (NiedersteOstholt et al. 2010)? What is the mass of the bound remnant? Is M54 the core of Sgr or simply aligned with the core by chance? Or has it sunk to the core from dynamical friction?

Indeed, even the distance to Sagittarius is somewhat uncertain, with most estimates clumping close to the initial $24 \mathrm{kpc}$ distance reported by Ibata et al. (1994) but ranging up to $26 \mathrm{kpc}$ (Monaco et al. 2004), 27 kpc (Layden \& Sarajedini 2000; Bellazzini et al. 2006; Kunder \& Chaboyer 2009; Sollima et al. 2010), or even $28 \mathrm{kpc}$ (Siegel et al. 2007, hereafter Paper IV).

Insight into some or perhaps all of these issues could be obtained by studying the three-dimensional orientation of the Sgr core and its incipient debris field. However, study of the Sgr core and its emerging tidal debris arms is hampered by their location behind a thick veil of foreground Milky Way bulge stars. Large photometric surveys (e.g., Ibata et al. 1994; M03) have shown the core to be elongated. However, these studies have not been able to resolve definitively the distance to the $\mathrm{Sgr}$ core, its three-dimensional shape, or the status of M54 as the core of Sgr or a chance alignment.

In this paper, we use data from the Advanced Camera for Surveys (ACS) of Galactic globular clusters (Sarajedini et al. 2007, hereafter Paper I) to overcome some of these difficulties and provide new insight into the spatial distribution of Sgr's inner regions. The high spatial resolution provided by the Hubble Space Telescope (HST) can overcome the crowding issues while the high precision of the deep ACS photometry can delineate faint photometric sequences that are undetectable from the ground. This was demonstrated by our study of the stellar populations in the Sgr core (Paper IV), which easily identified previously unseen features in the Sgr CMD.

Using the unique and powerful ACS data set, we now move beyond the Sgr center to examine the larger structure of the $\mathrm{dSph}$. In addition to four classical Sgr member clusters that are close to or in the Sgr core (M54, Terzan 7, Terzan 8, Arp 2; Da Costa \& Armandroff 1995), we have identified Sgr debris in the background of five Milky Way bulge clusters (Section 3.2). The fortuitous combination of a well-measured foreground cluster, a well-measured background population, and a previously determined age-metallicity relationship for Sgr (Paper IV) allows us to use these background features to measure precise relative distances for five additional lines of site. In combination with the member clusters, this allows us to probe the shape of the bound core and emerging Sgr tidal debris over a large solid angle. We compare the distances of Sgr member clusters, the distance of Sgr features in the background of Milky Way bulge clusters, and the density of the background Sgr material to the $N$-body model of tidal disruption from LM10a to gain new insight into the three-dimensional shape of Sgr and its globular cluster system, as well as what their properties reveal about the intrinsic shape, distance, and dynamical evolution of the dSph (Section 4).

\section{OBSERVATIONS AND DATA REDUCTION}

The ACS Survey of Galactic Globular Clusters (Paper I) is a photometric study of 65 nearby globular clusters using the Wide Field Channel (WFC) of the ACS aboard the HST. The objective is to use the high-precision color-magnitude diagrams (CMDs) provided by the HST/ACS/WFC to explore a host of issues in stellar evolution, cluster evolution, dynamics, and the formation of the Milky Way. To date, this program has provided detailed analyses of the CMDs of the NGC 1851, NGC 6366, and M54 globular clusters (Milone et al. 2008; Paust et al. 2009; Paper IV). We have also used the CMDs for global analyses of relative cluster ages (Marín-Franch et al. 2009, hereafter Paper VII), luminosity functions (Paust et al. 2010), horizontal branch morphologies (Dotter et al. 2010, hereafter Paper IX), and cluster centers (Goldsbury et al. 2010).

We observed our target clusters in the $F 606 W(\sim V)$ and $F 814 W(\sim I)$ filters with HST/ACS/WFC (Paper I). Photometry was generated through new point spread function (PSF) methods (Anderson et al. 2008, hereafter Paper V) and Vega-calibrated using the charge-transfer efficiency corrections of Riess \& Mack (2004), calibration procedures in Bedin et al. (2005), and zero points of Sirianni et al. (2005) with zero-point corrections updated to those of Bohlin (2007). The photometry of isolated saturated stars on short exposures was salvaged by summing all associated charge - a procedure previously applied by Gilliland (2004). For our clusters, the data pipeline provides 12 mag of precise photometry from nearly the tip of the red giant branch (RGB) to several magnitudes below the main-sequence turnoff (MSTO). Uncertainties are approximately $1 \%$ at $V \sim 22$ and $10 \%$ at $V \sim 25$. We have cleaned the photometric catalogs of non-stellar objects and poorly measured stars using trends of quality of fit against magnitude to select sources with the most star-like profiles and $<10 \%$ of the flux in their PSF aperture from other stars (see Paper V). The photometric data are available to the public from our archive. ${ }^{13}$

\section{DISTANCE MEASURES IN THE SGR FIELD}

\subsection{Sagittarius Member Clusters}

Table 1 lists the four Galactic globular clusters that are within a few core radii (given in M03 as 3.8) of Sgr. For each cluster, we list coordinates, in degrees, in the Sgr coordinate system $\left(\Lambda_{\odot}, B_{\odot}\right)$ defined by the Euler angles given in Table 2 of M03, in which $\Lambda_{\odot}$ is defined as the angular distance along Sgr's orbit with positive $\Lambda_{\odot}$ in the direction of the trailing debris. $B_{\odot}$ is the lateral angle from the Sgr meridian. The Sgr coordinate system allows a more natural analysis of the Sgr features relative to the $\mathrm{dSph}$ itself than other celestial coordinate systems.

\footnotetext{
13 http://www.astro.ufl.edu/ ata/public_hstgc/databases.html
} 


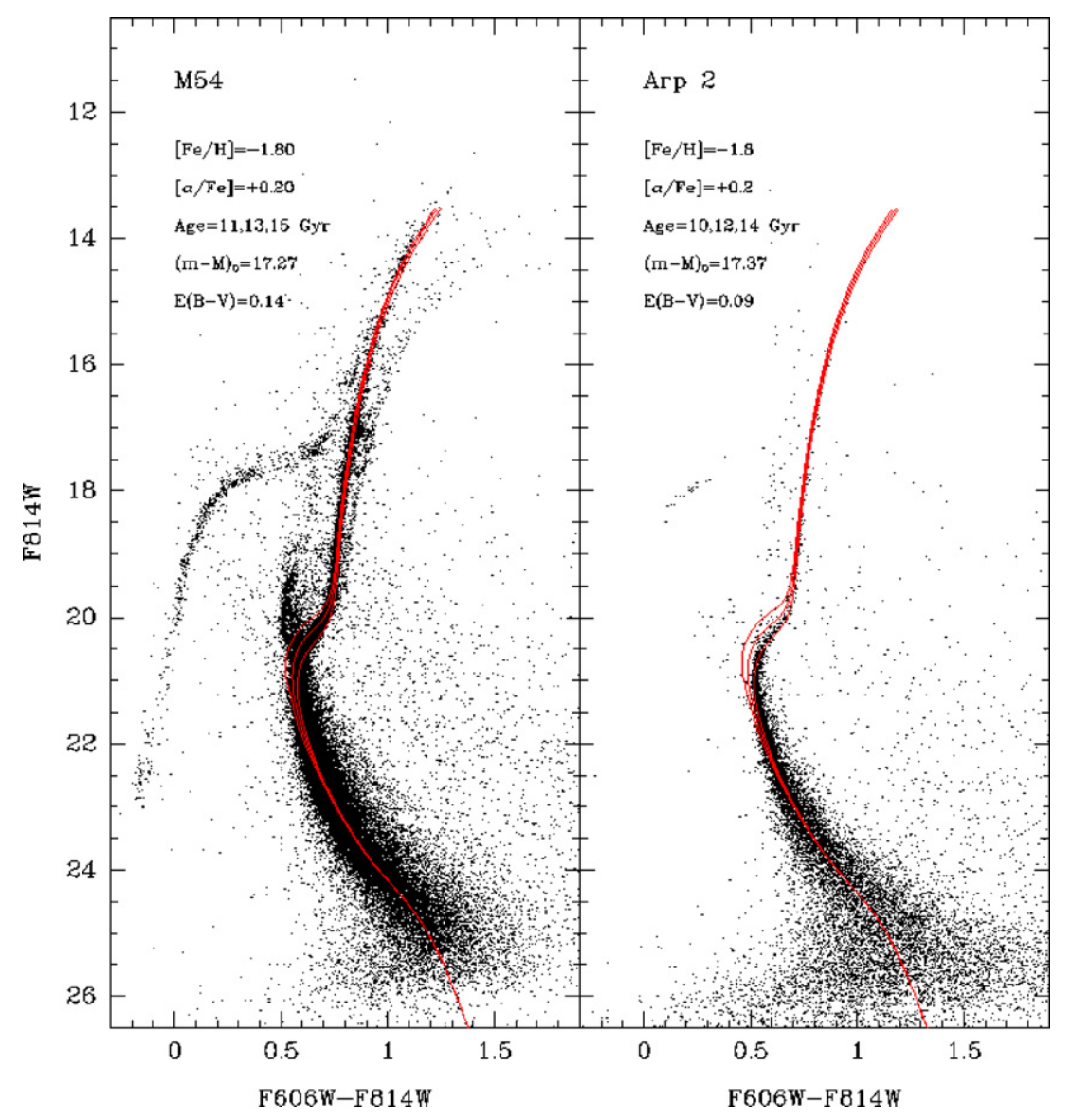

Figure 1. Isochrone fits to the clusters M54 and Arp 2 as derived in Paper IV and Paper IX, respectively. The isochrones and fits were adjusted slightly from Papers IV and IX to reflect recent spectroscopic abundance measures.

(A color version of this figure is available in the online journal.)

A number of other Galactic globular clusters have been suggested as members of Sagittarius (see, e.g., Dinescu et al. 2000; Palma et al. 2002; Bellazzini et al. 2002, 2003a, 2003b; Cohen 2004; Carraro et al. 2007; Carraro 2009). LM10b performed a robust analysis and concluded that, besides the classical members, Whiting 1 and NGC 5634 have a high probability of being part of Sgr and NGC 5053 and Pal 12 are moderately likely to be members. Pal 12 and NGC 5053 were included in the ACS survey. However, these clusters are situated $39^{\circ}$ and $266^{\circ}$ along the Sgr tidal stream (LM10b), respectively. While their distances could provide insight into the shape of Sgr's tidal arms, we have left them out of our analysis, which is focused on the three-dimensional orientation of the Sgr core.

Figures 1 and 2 show the CMDs for the classical Sgr clusters. For Terzan 7, Terzan 8, and Arp 2, we began our analysis with the isochrones fitted to the cluster sequences in Paper IX. These isochrones, described in detail in Dotter et al. (2007), were fitted in Paper IX based on initial metallicity estimates from the most recent catalog of Harris (1996, 2010 edition), tweaked slightly to better match the isochrones to the observed photometry. For this paper, we have adjusted the fits slightly to reflect the most recent $[\mathrm{Fe} / \mathrm{H}]$ and $[\alpha / \mathrm{Fe}]$ values measured for the Sgr clusters (see, e.g., Sbordone et al. 2005; Mottini et al. 2008), as listed in Table 1. In all cases, the distances are based on the model-predicted absolute magnitudes, scaled to an assumed value of the solar luminosity $\left(3.4818 \times 10^{33} \mathrm{erg} \mathrm{s}^{-1}\right)$. Extinction values were determined directly from the isochrone fits and converted into $E(B-V)$ values from the $H S T$ colors using the coefficients of Paper I. Distance uncertainties are difficult to estimate because they depend upon both the mass-metallicity-luminosity relationship of the stars and the bolometric corrections. We estimate that the absolute distance uncertainties are approximately $0.1 \mathrm{mag}$ while the relative distance uncertainties, which are the more important to our differential analysis, are 0.05 mag or lower.

Paper IX did not analyze M54 due to the multiple populations present in the field. Paper IV derived a distance/reddening based on the dominant metal-poor population of M54 itself. As noted in Section 4.4, there are some differences between the semi-empirical isochrones used in Paper IV and the synthetic isochrones used in Paper IX and this paper. However, these differences mostly affect the youngest Sgr populations. Using the most recent synthetic isochrones, we find that the distance/age/abundance/reddening used in Paper IV still provide an excellent fit to the photometry of the dominant metal-poor main sequence (see Figure 1).

In an effort to detect any diffuse background Sgr tidal debris influencing our analysis of the clusters near the center of Sgr, we took the cluster fiducials for Arp 2, Terzan 7, and Terzan 8 from Paper VII and shifted them in magnitude, counting the number of stars within $0.05 \mathrm{mag}$ of the fiducial. For a simple stellar population, like that expected in a typical globular cluster, we would expect the count of stars to follow a Gaussian pattern, albeit with a slight increase at faint magnitudes from increasing photometric scatter near the fiducial and a tail at bright magnitudes due to unresolved binary stars. If 


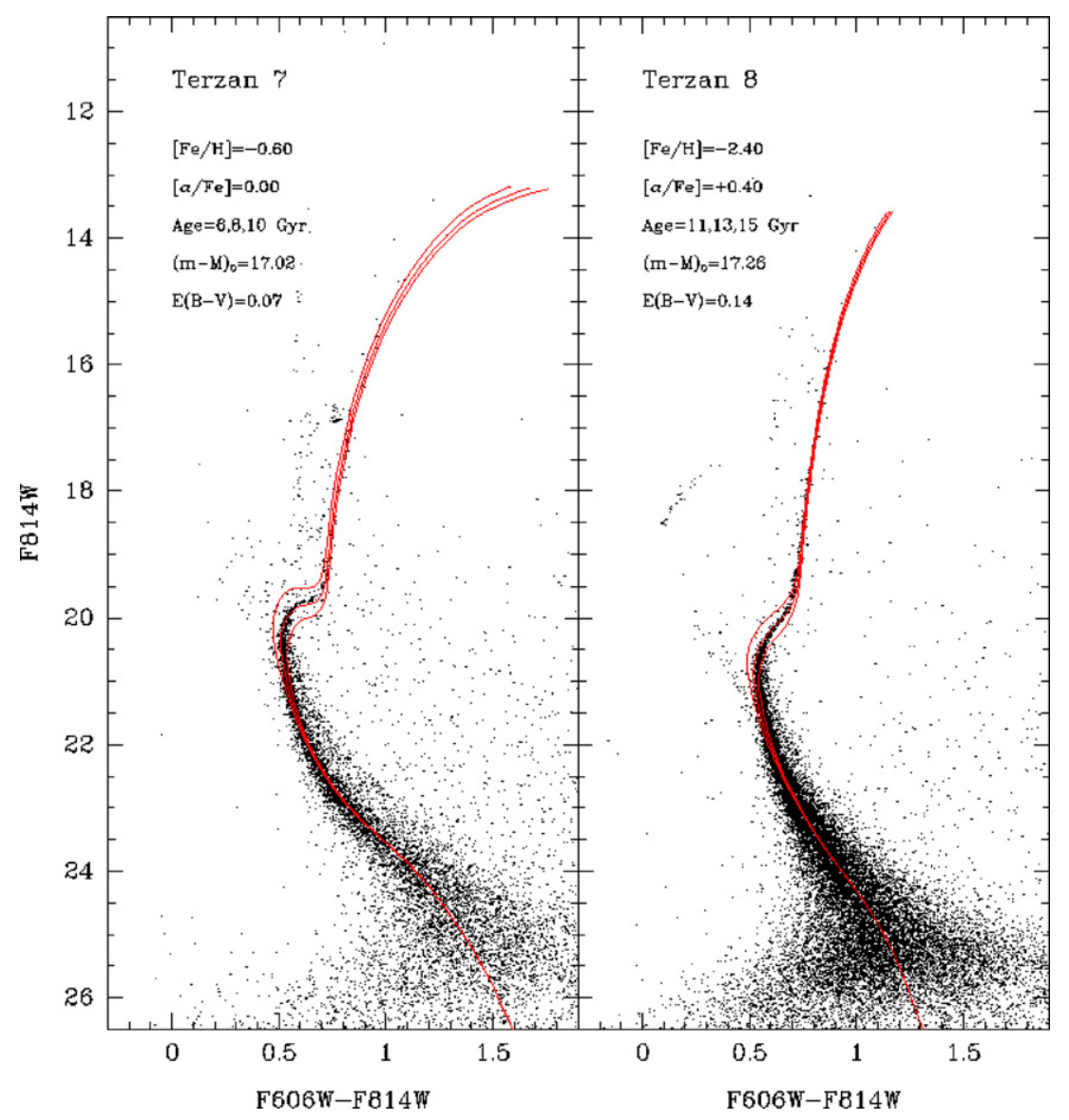

Figure 2. Same as Figure 1 for Terzan 7 and Terzan 8.

(A color version of this figure is available in the online journal.)

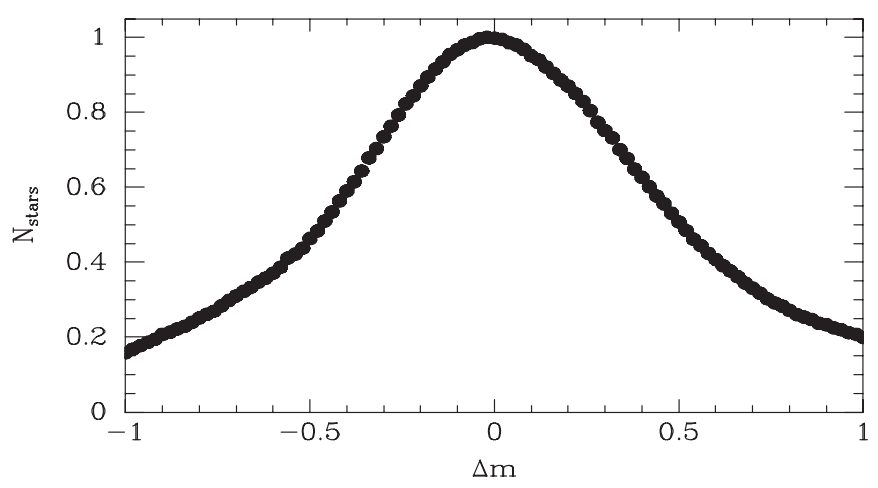

Figure 3. Normalized number of stars within 0.05 mag of the Paper VII fiducial as a function of a magnitude shift applied to the fiducial. This shows the test applied to Terzan 7; the results for Arp 2 and Terzan 8 are similar.

a significant second population at one distance-in this case Sgr-were present, it might show up as a second bump or as an asymmetry in the distribution, even if the fiducial does not precisely match the background population. Detecting the Sgr background feature would allow us to constrain any effect it has upon the isochrone fitting and measure any distance discrepancy between the Sgr member clusters and the stellar tidal debris stream.

For Arp 2, Terzan 7, and Terzan 8, the number counts follow a Gaussian distribution with no deviation larger than the Poisson noise (see Figure 3). This indicates either (1) that the clusters are in or near the mean distance of the Sgr stream; (2) that the Sgr background population is simply too diffuse; and/or (3) too close in color-magnitude space to the primary cluster sequence to show up distinctly in our analysis. Given that the distance measures in Table 1 suggest a significant distance discrepancy for at least Terzan 7, (2) is the more likely explanation.

\subsection{Sagittarius Background Features}

In the course of examining the CMDs of the ACS survey globular clusters, we identified five bulge cluster CMDs that appear to contain a faint secondary stellar sequence parallel to and below the cluster main sequence (Figure 4). This secondary sequence is too bright and red to represent the white dwarf sequence of the cluster and has the appearance of a diffuse main sequence. It is broader than the photometric uncertainties would indicate for a simple stellar population, which hints at a composite population. Given that the suspect clusters lie in the foreground of the body and tidal stream of Sgr, it is likely that this feature represents the diffuse Sgr tidal stream in the background of these clusters. This Sgr background feature has been hinted at before (see, e.g., Mateo et al. 1996) but has not been depicted as cleanly as shown in Figure 4. The deep and precise ACS data delineate three magnitudes of this secondary sequence. This feature appears in all of the ACS survey clusters that are relatively close (within $11^{\circ}$ ) to the Sgr core.

The fortuitous alignment of the Sgr background features with simple and well-measured foreground clusters - in combination with the consistent and precise photometry produced by our pipeline-provides an unprecedented opportunity to measure the distance of the Sgr core and/or tidal stream along multiple lines of sight across the face of the dSph. While the foreground 

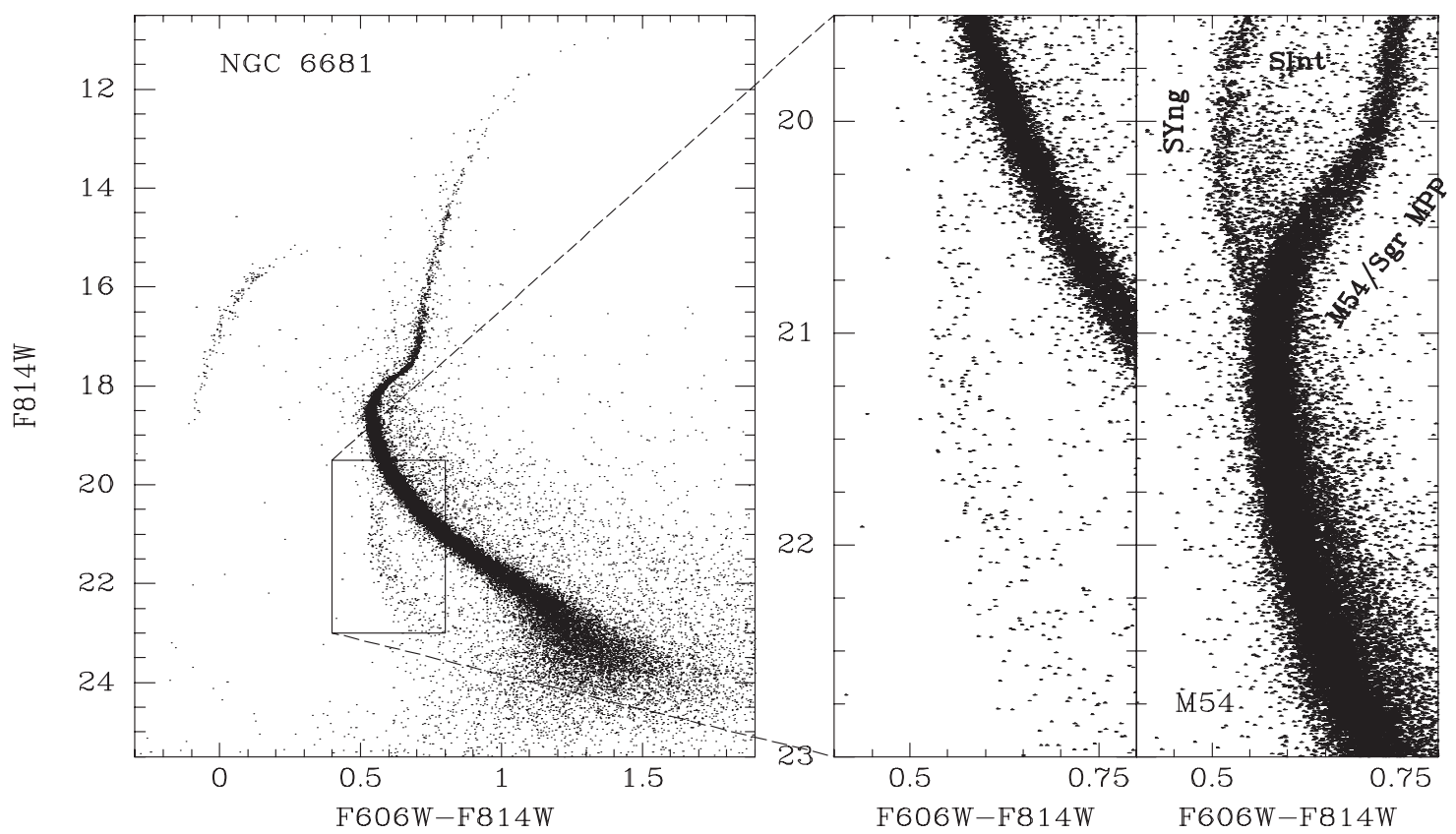

Figure 4. Background Sgr feature, as discovered in the field of NGC 6681. The middle panel shows a zoomed-in view of the second MS in the background of the bulge cluster. The right panel shows a similar region of the CMD of M54-which either is at the same distance or slightly in the foreground of the Sgr core-shifted $0.15 \mathrm{mag}$ in accordance with the distance measured in Table 2. We have labeled the young (SYng) and intermediate (SInt) Sgr populations in M54's core, as defined in Paper IV and in the text. The metal-poor population, which creates the dominant MSTO/SGB, is a mix of M54's metal-poor population and Sgr's and is labeled appropriately (M54/Sgr MPP). Note the similarity between the background population of NGC 6681 and the intermediate-age populations of Sgr (which tend to be on the redward side of the broad M54/Sgr main sequence). Note also that the SYng population—-the brightest and bluest Sgr feature-is not seen in the Sgr background feature, indicating that this population is confined to the Sgr core. The breadth of the Sgr feature in the NGC 6681 field is not the product of observational uncertainty but reflects genuine dispersion in the Sgr stellar populations.

clusters generally have different abundances than typical Sgr core stars, the stars of the foreground MSs provide a useful first estimate of the relative distances to the suspected Sgr features. Using the fiducials from Paper VII, we measured by eye the color-magnitude shifts needed to move each foreground cluster's fiducial to overlap the suspected Sgr population. These relative measures confirm that the secondary sequences are at a distance consistent with that of the Sgr core.

We then refined these rough estimates by measuring the relative distance of each of the background features to each other. We first removed from each CMD those stars that lay within $0.1 \mathrm{mag}$ of the foreground clusters' fiducial, as defined in Paper VII. We then established NGC 6681, which has the strongest Sgr feature, as a template and overlaid the CMD of NGC 6624, which has the second strongest. We shifted the CMD of NGC 6624 in color-magnitude space by eye until the Sgr feature of NGC 6624 overlapped that of NGC 6681. The data from the two clusters were then combined and the cluster with the next-strongest feature overlaid and shifted. This process continued through all five of the bulge clusters with clean secondary sequences until color-magnitude shifts were established for the entire sample. This CMD shifting and adding enhances the Sgr feature (see Figure 5) and allows us to re-shift each cluster iteratively in the combined CMD to further refine the relative distance estimates.

These relative measures were then calibrated to absolute distance by overlaying the isochrones fitted to the Sgr population from Paper IV. The M54/Sgr field is a complex medley of populations. Paper IV describes the Sgr field as a composite of seven populations (see their Figure 2), the most prominent of which are shown in Figure 4.

1. SVYng: a diffuse very young (100-800 Myr) population of super-solar abundance.

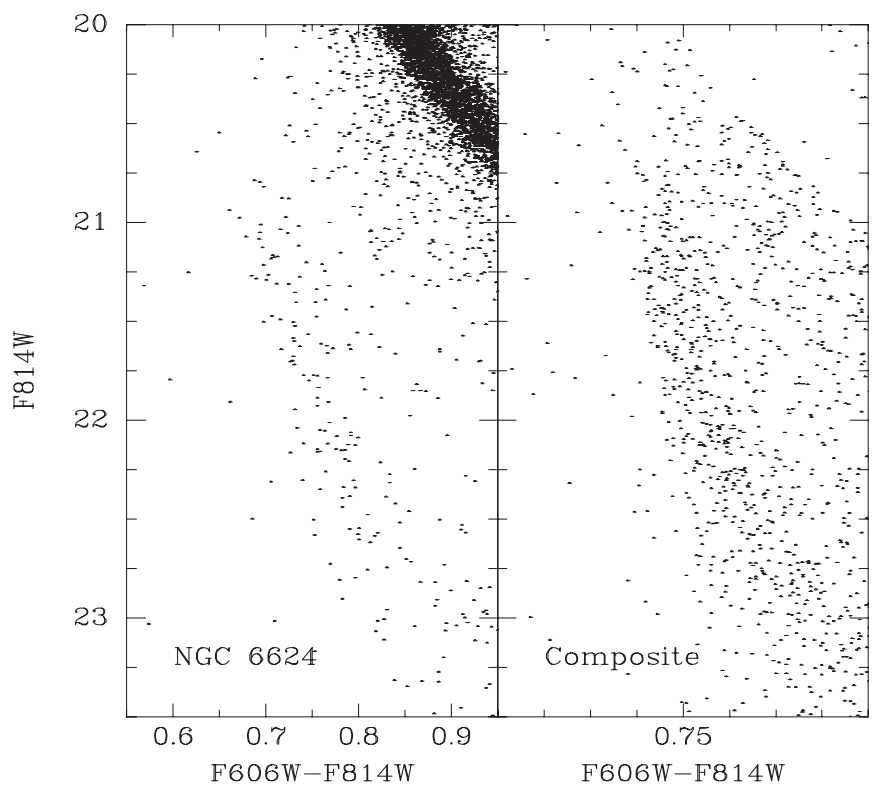

Figure 5. Enhancement of the Sgr background feature. The left panel shows the CMD for NGC 6624. The right shows the composite CMD of all five background features, shifted to the same distance and reddening as the NGC 6624 background feature.

2. SYng: a young (2.3 Gyr) population of solar abundance. This population could be of slightly younger age $(1.75 \mathrm{Gyr})$ if it has an enhanced He abundance similar to the young population identified in $\omega$ Centauri (Carretta et al. 2010b).

3. SInt: three intermediate-age (4-7 Gyr) populations of intermediate abundance $([\mathrm{Fe} / \mathrm{H}]=-0.3$ to -0.7$)$. 
Table 2

Bulge Clusters with Sgr Background Features

\begin{tabular}{lrccccccc}
\hline \hline Cluster & $\begin{array}{c}\Lambda_{\odot} \\
(\mathrm{deg})\end{array}$ & $\begin{array}{c}B_{\odot} \\
(\mathrm{deg})\end{array}$ & $(m-M)_{0, \text { clus }}$ & $E(B-V)_{\text {clus }}$ & $(m-M)_{0, \text { Sgr }}$ & $E(B-V)_{\text {Sgr }}$ & $N_{\text {Sgr }}$ & $\begin{array}{c}D_{\text {Sgr }} \\
(\mathrm{kpc})\end{array}$ \\
\hline NGC 6624 & 353.4 & 2.8 & 14.58 & 0.26 & 17.33 & 0.31 & 155 & $29.2 \pm 0.7$ \\
NGC 6637 & 355.4 & 4.3 & 14.75 & 0.17 & 17.35 & 0.22 & 113 & $29.5 \pm 0.7$ \\
NGC 6652 & 356.5 & 4.8 & 14.84 & 0.12 & 17.42 & 0.17 & 62 & $30.5 \pm 0.7$ \\
NGC 6681 & 357.9 & 3.7 & 14.87 & 0.10 & 17.42 & 0.14 & 222 & $30.5 \pm 0.7$ \\
NGC 6809 & 9.7 & 0.8 & 13.67 & 0.12 & 17.34 & 0.16 & 84 & $29.4 \pm 0.7$ \\
\hline
\end{tabular}

4. Sgr MPP: an old $(11 \mathrm{Gyr})$ metal-poor $([\mathrm{Fe} / \mathrm{H}]=-1.3)$ population that significantly overlaps M54's metal-poor population.

5. M54 MPP: the old metal-poor population of M54 itself $(13 \mathrm{Gyr},[\mathrm{Fe} / \mathrm{H}]=-1.8)$, which significantly overlaps the Sgr MPP.

The analysis in Paper IV has since been complemented by the spectroscopic survey of Carretta et al. (2010b), whose spectroscopic metallicity distribution is similar to that described above (see their Figure 1). However, they describe the M54 MPP population as having a slightly higher metallicity of $[\mathrm{Fe} / \mathrm{H}]=-1.55$ with a significant $(0.19 \mathrm{dex})$ dispersion. $\mathrm{P}$. Frinchaboy et al. (2011, in preparation) explore the metallicity distribution along Sgr's major and minor axes and show clear evidence of a spatial trend in abundance (about 0.2-0.3 dex out to the radii of our bulge cluster fields). This trend could be critical to analysis of the Sgr background features.

The derived absolute distance of the Sgr CMD features, as measured by comparison to the Paper IV isochrones, is sensitive to which of these seven populations are included in the analysis. If we include all seven populations, the inferred distances would be many kiloparsecs farther than the distances measured for the Sgr core clusters. However, including all seven populations is likely inappropriate for the background features, which all lie outside of the core of Sgr (defined as 3.7-3.9 in M03) and are well away from the central M54 pointing. No study, from either the ground or the $H S T$, has found the youngest populations seen in the analysis in Paper IV beyond these innermost regions of Sgr, and none of the five background features have the bright blue SYng population seen in the M54 field (the brightest turnoff in Figure 1).

The aforementioned spectroscopic studies of Sgr's inner regions (Carretta et al. 2010b; P. Frinchaboy et al. 2011, in preparation) would favor using the more metal-poor of Sgr's seven populations. The Frinchaboy et al. study, in particular, traces out the major axis of Sgr into regions overlapping our survey. The metallicity distribution in their outer regions would be best matched by a combination of the Sgr MPP population with the more metal-poor SInt populations (those with $[\mathrm{Fe} / \mathrm{H}]$ of -0.5 and -0.7$)$. Such a choice of isochrones would also be consistent with the approximately 0.2 dex drop in metallicity between the core and the tidal arms indicated by studies of the tidal arms (LM10a; Alard 2001; Chou et al. 2007, 2010; Giuffrida et al. 2010). We therefore have chosen these three populations - Sgr MPP and the two poorest SInt's-to represent the background Sgr debris. The uncertainty over which populations to use (and the relative strengths of each) introduces some uncertainty into our distance measures. This uncertainty is estimated to be around 0.05 mag based on a comparison of the distance moduli derived for various combinations of stellar populations.
The isochrones were first placed at M54's distance and NGC 6681's reddening, with the $A_{F 606 W}$ and $A_{F 814 W}$ extinction coefficients set to those of Sirianni et al. (2005). We then varied the distance and reddening until the Sgr isochrones overlapped the composite background population created from the shifted and co-added CMDs of all five clusters. To check internal consistency, we then fitted the isochrones to the background population of each cluster CMD on an individual basis. We recover the relative shifts between the different background features to a consistency of approximately $0.03 \mathrm{mag}$.

Figures 6-10 show both the Hess diagrams of the CMDs and the unbinned CMDs of the foreground clusters with the fitted Sgr isochrones overlaid. The parameters of the fits are listed in Table 2 and include coordinates in the Sgr system, the distances, and reddenings to the foreground clusters, and the distances and reddenings to the Sgr CMD features. $E(B-V)$ values are calculated from $E(F 606 W-F 814 W)$ using the coefficients of Paper I. We estimate, for each field, that the relative distance modulus uncertainty is $0.05 \mathrm{mag}$ and the reddening uncertainty is $0.01 \mathrm{mag}$. The absolute distance uncertainty is larger, depending on the absolute calibration of the isochrones. Table 2 also lists a value for $N_{\mathrm{Sgr}}$, a crude measure of the strength of the Sgr feature. It is simply the number of stars in the magnitude range $21<F 814 W_{0}<23$ that are within $0.1 \mathrm{mag}$ (quadrature of color and $F 814 \mathrm{~W}$ magnitude) of one of the three most metal-poor Sgr isochrones. The final column lists the linear distance to the Sgr CMD features in kpc.

As may be seen, the distances of the Sgr CMD features are similar to those of the Sgr core and associated globular clusters. All of the Sgr CMD background features show a slightly increased reddening compared to their respective foreground clusters of $0.02-0.05 \mathrm{mag}$ in $E(B-V)$. On average, the Sgr CMD features are best fit with 0.04 mag additional reddening before that of the foreground cluster and 0.02 mag more reddening than the values estimated from the reddening maps of Schlegel et al. (1998). The additional color shift compared to the foreground cluster could reflect additional reddening along the line of sight. The foreground clusters are all within 5-9 kpc of the Sun and all have Galactic $Y$ and $|Z|$ coordinate values of less than 1 and $2 \mathrm{kpc}$, respectively (with $X Y Z$ centered on the Sun, $Y$ defined along the direction of Galactic rotation, and $Z$ defined as distance above the plane). While the $|Z|$ distances are large compared to the scale height of the dust at the solar radius ( $\sim 134 \mathrm{pc}$; Marshall et al. 2006), the dust scale height is expected to be larger in the Galactic bulge. Even a small amount of additional dust from the bulge could produce the required additional reddening (although we note that all five clusters are close to the total column reddenings given in Schlegel et al. 1998).

The additional shift in color is required to provide an adequate isochrone fit to the background features. Figure 11 shows NGC 6681 with the Sgr isochrones overlaid at the canonical Sgr 


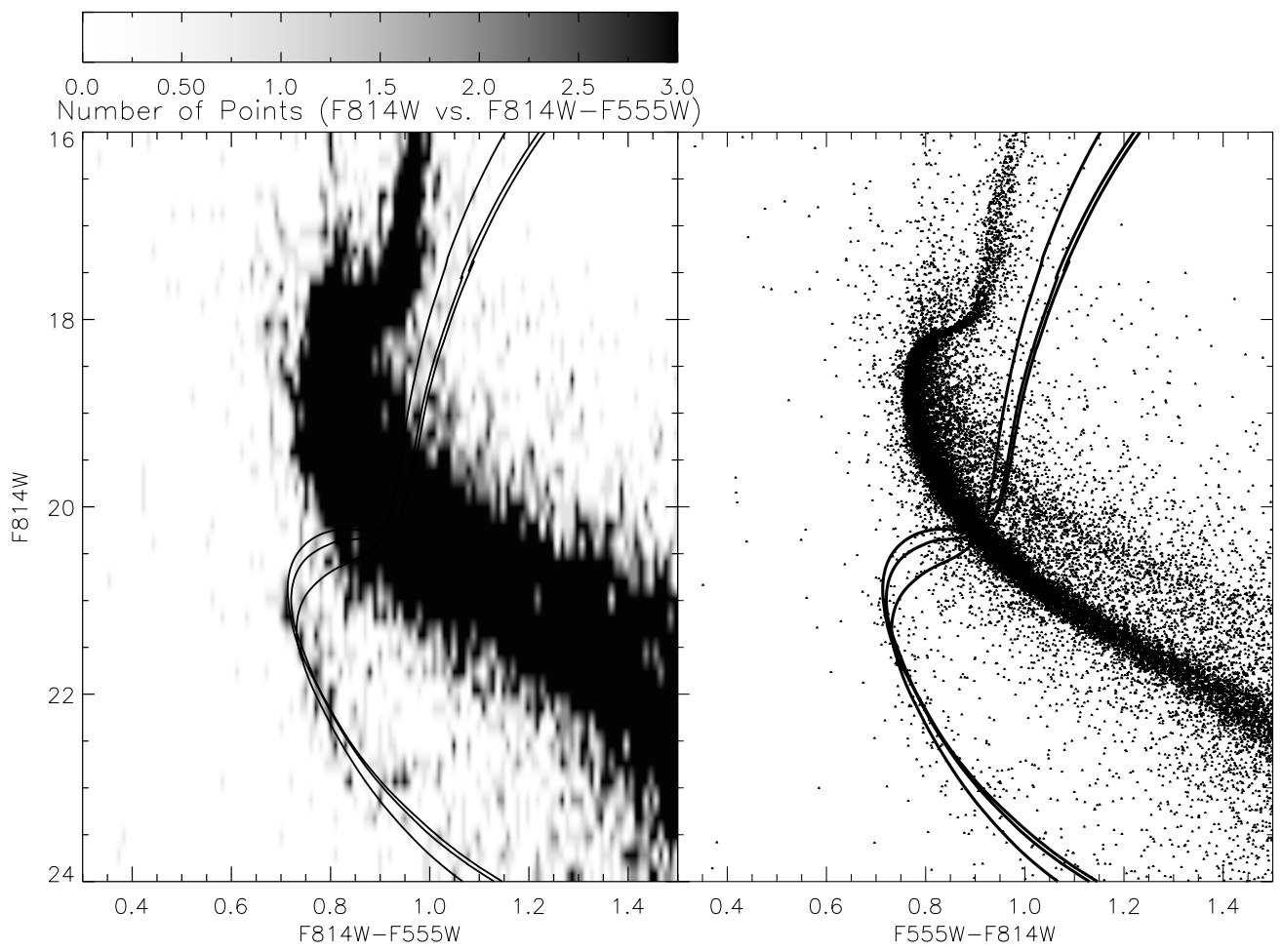

Figure 6. Constraining the background Sgr feature in the NGC 6624 bulge cluster field. The left panel shows a logarithmic Hess diagram with the scale stretched to provide maximum contrast for the Sgr background feature. This has the effect of saturating the foreground cluster. The right panel shows the unbinned CMD. The isochrones are an overlay of the adopted SInt and Sgr MPP populations used to fit the HST/ACS CMD of the Sgr core in Paper IV, shifted to the reddening and distance of the Sgr feature from the field as given in Table 2.

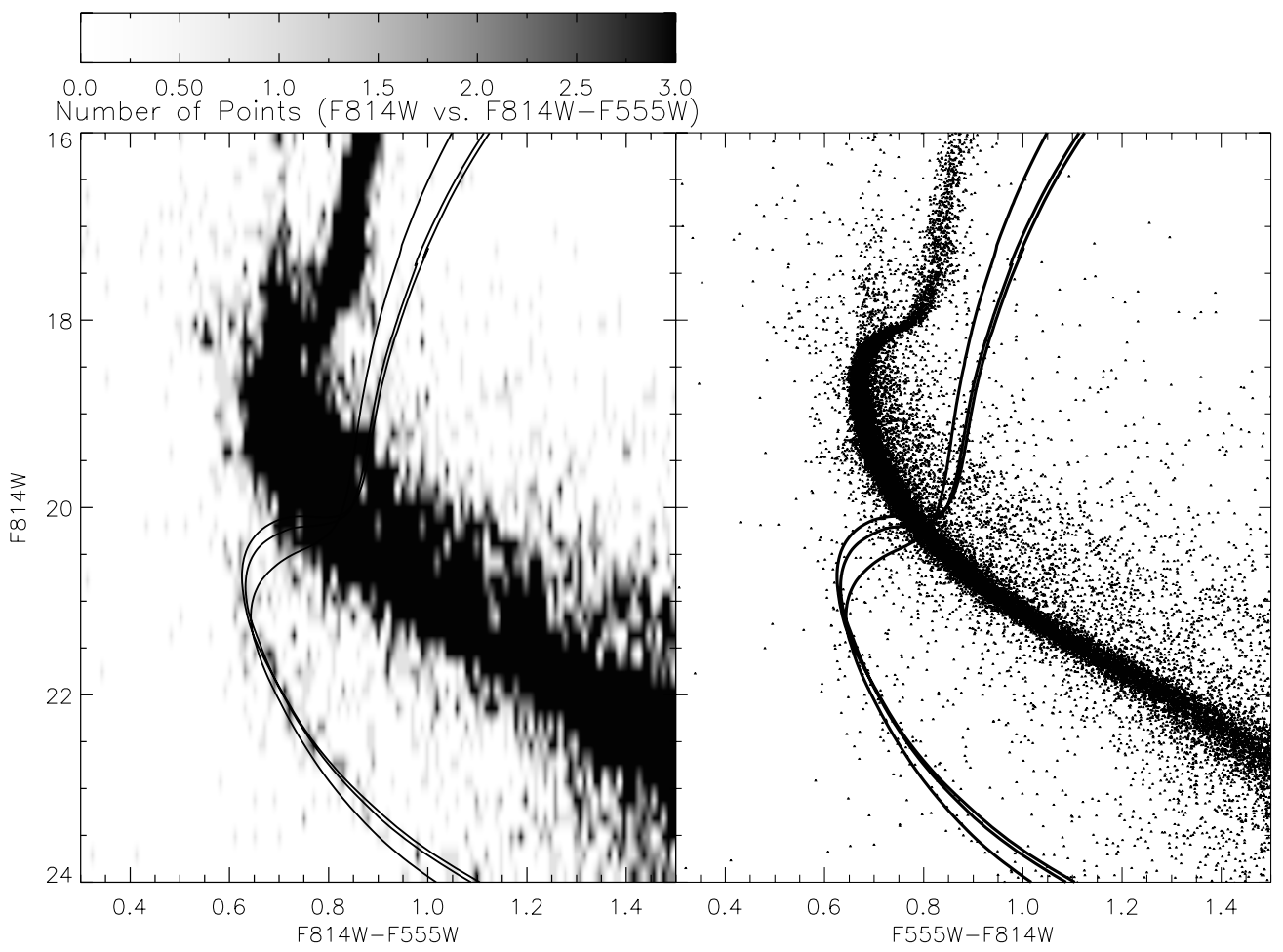

Figure 7. Constraining the background Sgr feature in the NGC 6637 bulge cluster field. See Figure 6 for description.

distance and NGC 6681's foreground reddening. The isochrone MSTO is notably brighter and bluer than the observed MSTO and the MS of the isochrone does not overlap the observed MS at all. Shifting the isochrone to the observed MS with a pure magnitude shift would require the Sgr feature to be $0.3 \mathrm{mag}$ closer along the line of sight and do a poor job of recreating the slight upward turn of the brighter portion of the MS just before the turnoff.

An alternative explanation for the additional measured reddening is that we have misinterpreted a change in the turnoff 


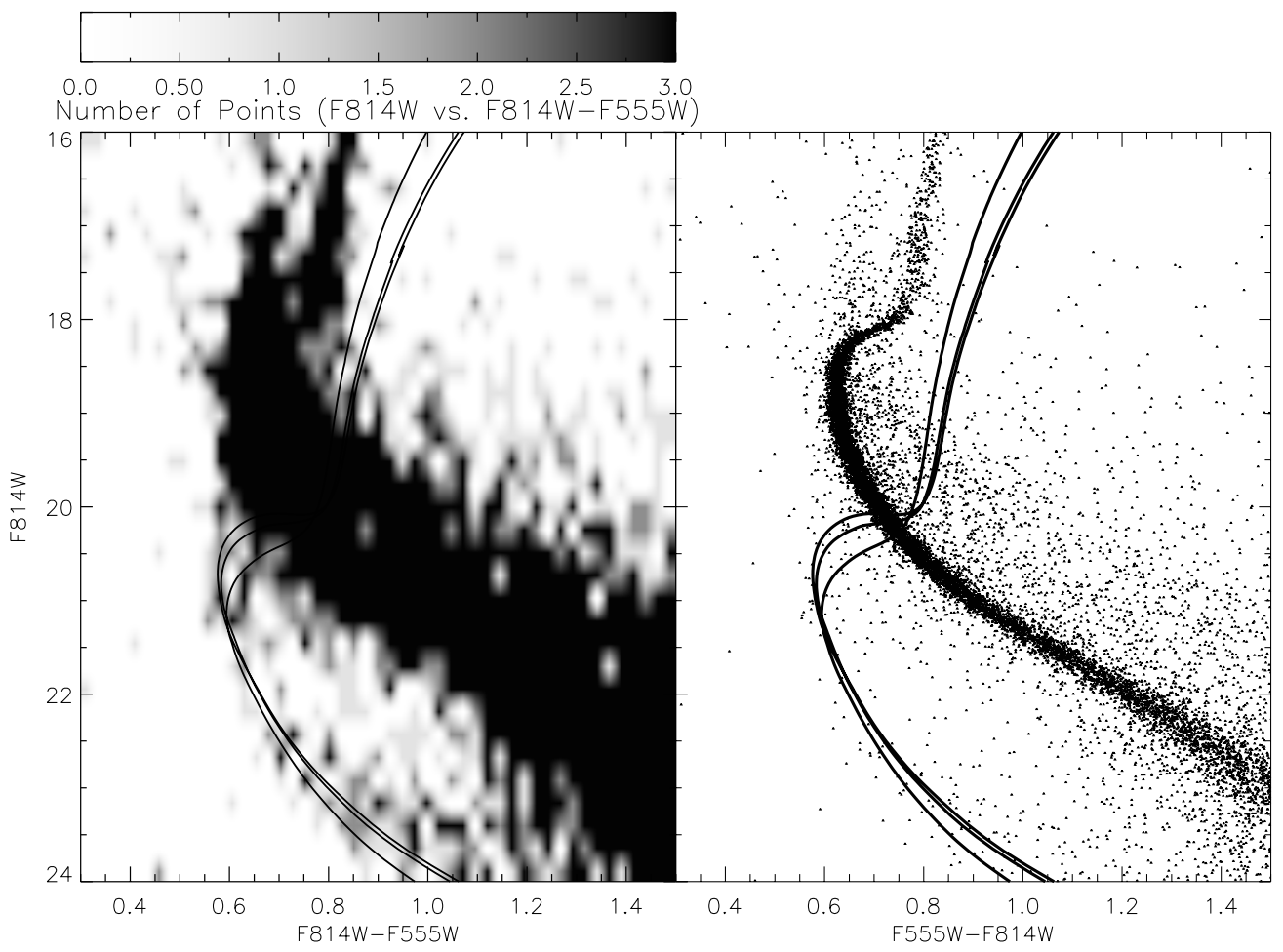

Figure 8. Constraining the background Sgr feature in the NGC 6652 bulge cluster field. See Figure 6 for description.

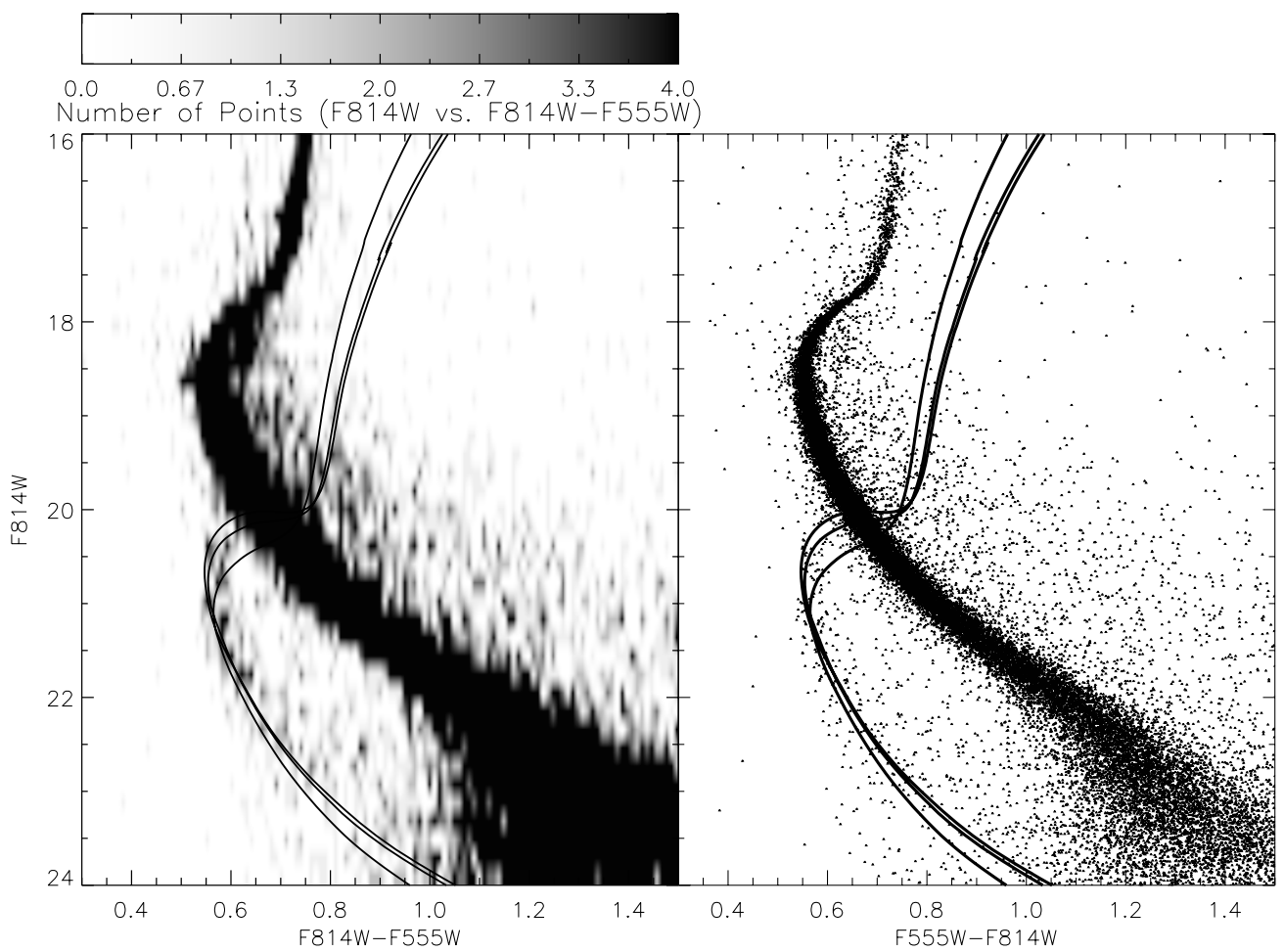

Figure 9. Constraining the background Sgr feature in the NGC 6681 bulge cluster field. See Figure 6 for description.

color over the extended distribution of Sgr as additional reddening. Such a difference in turnoff color would require the extended Sgr population be either older or more metal-rich than the Sgr core population. The latter is unlikely, given the known metallicity gradients. However, the former-an age difference-remains a possibility. Our analysis explicitly includes an age differential in Sgr by using only the older isochrones from Paper IV in our analysis of the background features. Paring down the Sgr background population to only the oldest SInt population and Sgr MPP from Paper IV would move the background features closer by approximately $0.05 \mathrm{mag}$ in distance modulus. Stripping the Sgr stream population down to only Sgr MPP would move the background features closer by another 0.14 mag. However, neither of these changes would 


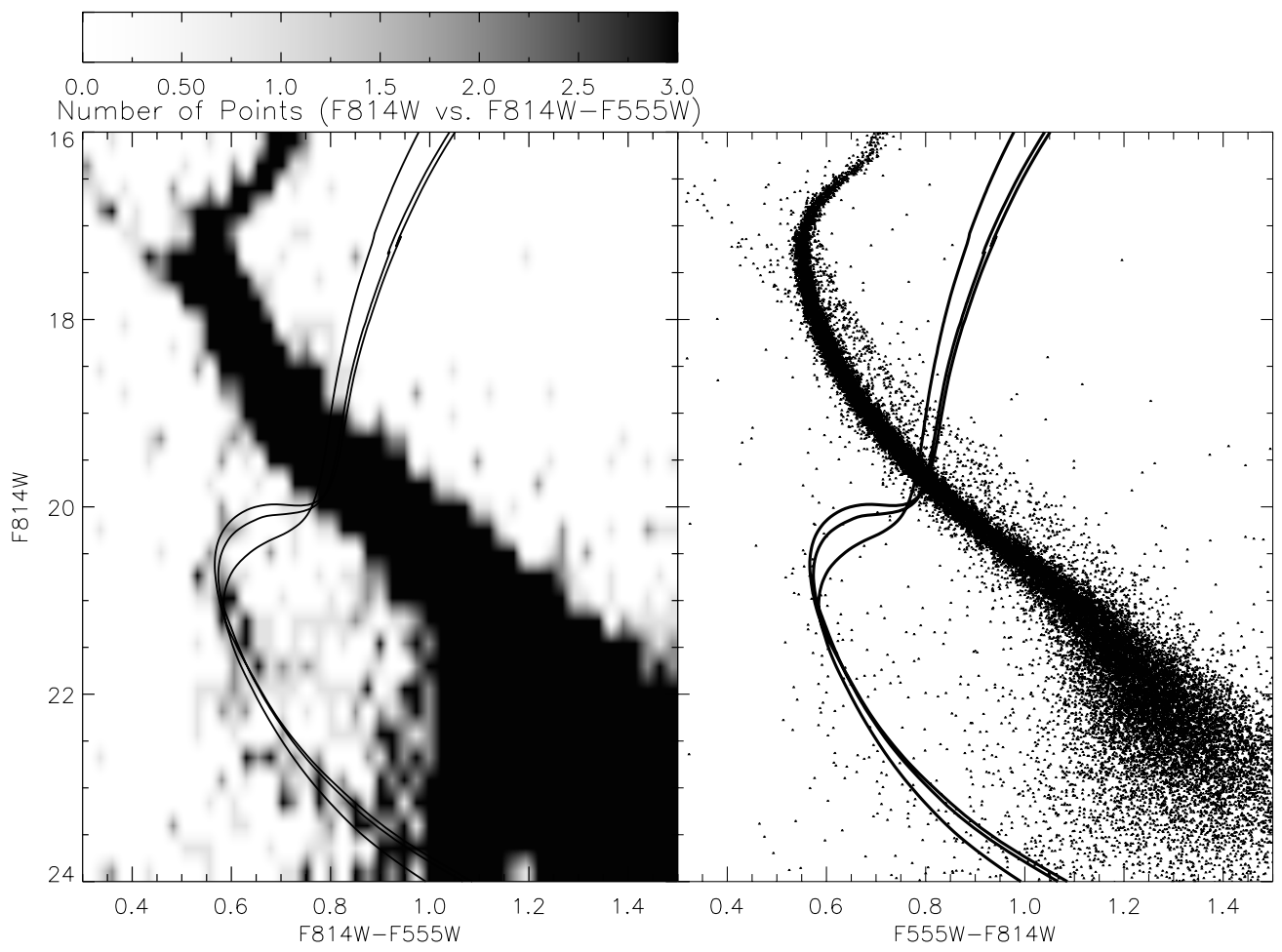

Figure 10. Constraining the background Sgr feature in the NGC 6809 bulge cluster field. See Figure 6 for description.

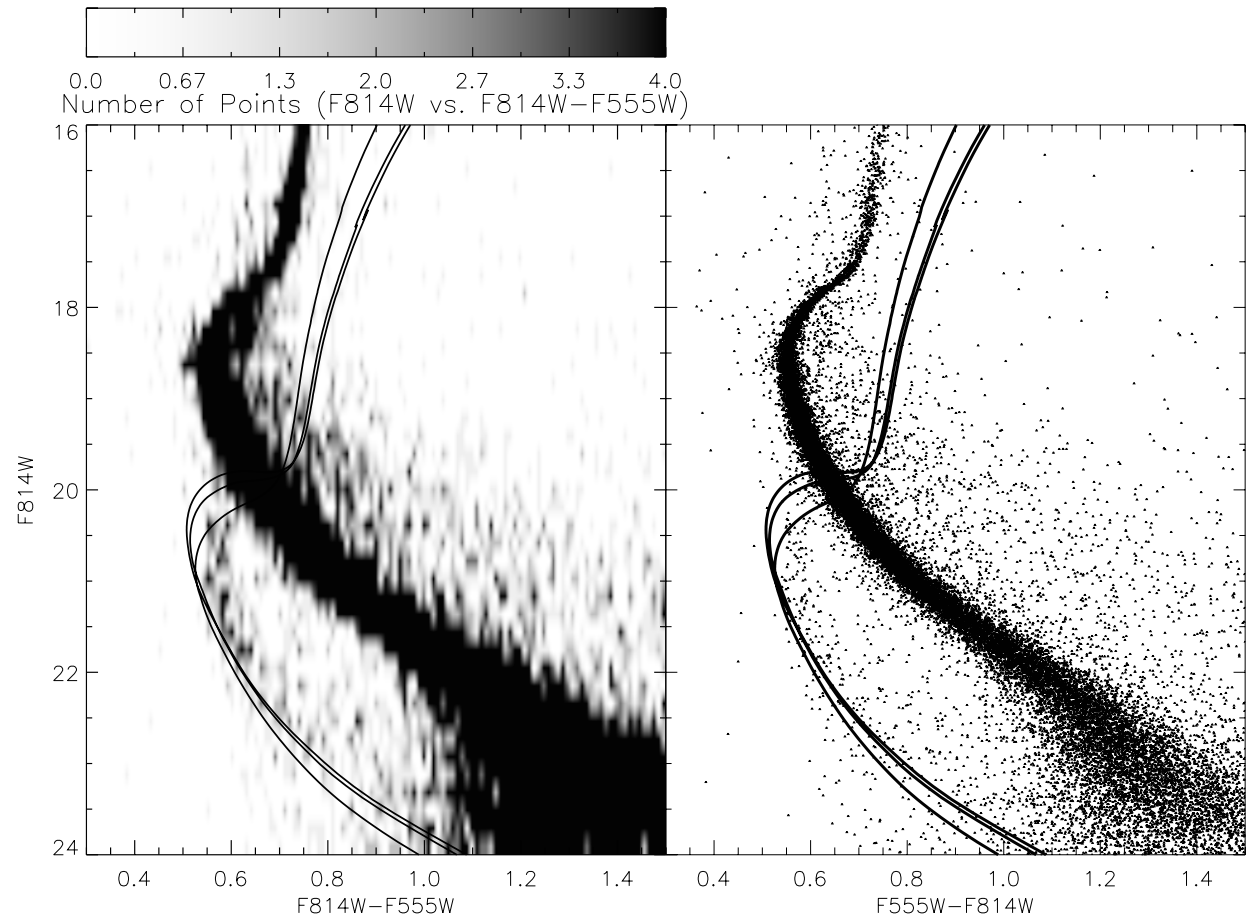

Figure 11. Modified version of Figure 9 in which the Sgr isochrones are set to the reddening of the foreground cluster and the distance of M54. Note that the isochrones miss the MSTO badly without the additional reddening.

remove the readily apparent reddening difference between the foreground bulge cluster and background Sgr feature, because the older populations are also more metal-poor and therefore have a bluer MS. Moreover, a single population would result in an Sgr background feature far narrower in color-magnitude space than that seen in the CMDs-either in our study or the recent HST study of Pryor et al. (2010) - and conflicts with the abundance distribution observed by P. Frinchaboy et al. (2011, in preparation). Finally, stripping down the Sgr field popula- tion to its oldest components would result in a metallicity for the extended Sgr dSph that is lower than that measured in the extended streams by Alard (2001) and Chou et al. (2007). We therefore find it unlikely that the difference in apparent foreground reddening is a mistaken analysis of a difference in stellar populations.

A final explanation is that systematic error in the models and synthetic (or even empirical) colors is masking itself as a difference in reddening, i.e., that we are measuring a discrepancy 


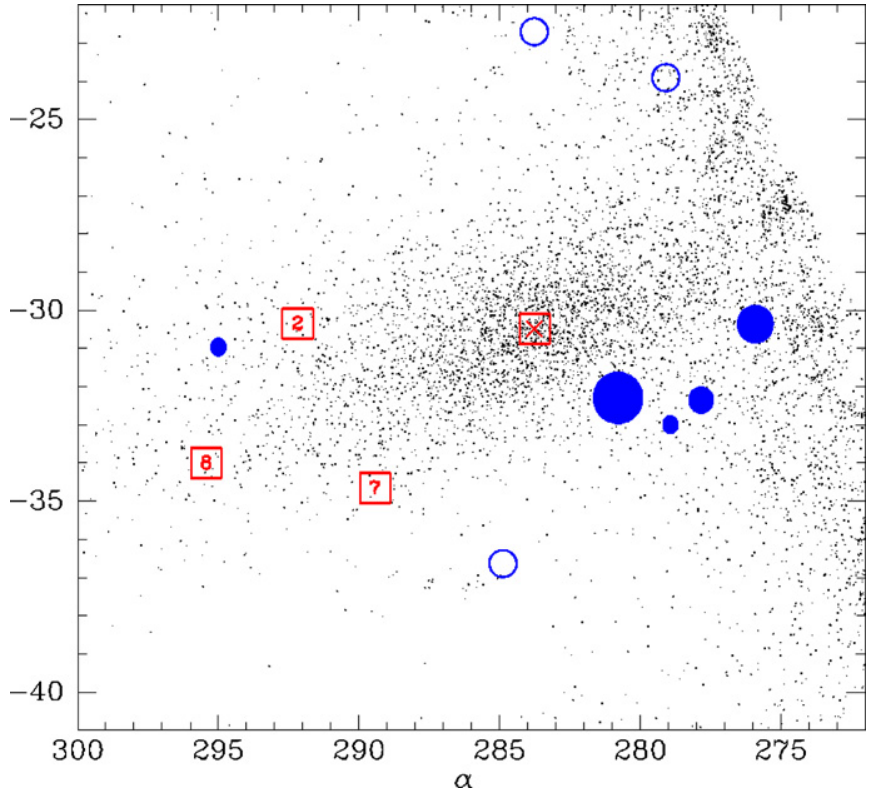

Figure 12. ACS survey detections of Sgr clusters and Sgr field stars seen in the ACS globular cluster survey fields as background debris compared to the M-giant map in the area around the Sgr dSph by Majewski et al. (2003). Red squares indicate Sgr globular clusters while filled blue circles are Sgr detections as background features to Milky Way globular clusters, with point size indicating the strength of the Sgr detection. Open blue circles are three clusters in the ACS survey program that do not have Sgr CMD features.

(A color version of this figure is available in the online journal.)

in the isochrones rather than a discrepancy in the reddening. We find this unlikely, given the range of abundances in the foreground clusters $(-0.44$ to -1.94$)$ and the success the isochrones have had in reproducing the color-magnitude shapes of the full sample of clusters in Paper IX.

In the end, however, our analysis is based on the measures of Sgr features relative to each other, not to the foreground clusters. So while a problem in reddening or photometry may change the absolute values of our measurements, it will have no effect on the relative measures. This is particularly germane to NGC 6681, which has the strongest Sgr feature and a color shift identical to that of the M54 central field. We are comparing the same isochrones to the same populations with the same color locus, minimizing any differential problems with our calibration or analysis.

\section{THE THREE-DIMENSIONAL ORIENTATION OF THE SAGITTARIUS dSph}

Previous investigations of Sgr establish a baseline against which to compare and contrast our new Sgr distance and density measures. In this section, we compare the new CMD detections to both previous observational data and theoretical models.

Figure 12 shows the positions and densities of our $\mathrm{Sgr}$ detections compared to the plot of $\mathrm{M}$ giants from M03. The open squares represent the Sgr clusters while the solid points are the background features, with the size reflecting the relative density of the Sgr detections as measured by $N_{\text {Sgr }}$ in Table 2 . Open circles represent ACS survey targets that do not have Sgr CMD features in them. We have not used the M-giant distances for our analysis because they are highly uncertain (typical $\sigma_{d} / d \sim 0.2-0.25, \mathrm{M} 03$ ), are on a different distance scale than the ACS photometry, and are calibrated to a single color-magnitude relation that may not adequately reflect the abundance gradient within Sgr that must be accounted for to precisely probe Sgr's three-dimensional shape.

Figure 13 shows the positions and distances of our Sgr clusters and background features compared with two variations of the LM10a $N$-body model of Sgr disrupting in the Milky Way potential, one with $\mathrm{Sgr}$ at a distance of $28 \mathrm{kpc}$ along the line of sight and the other with Sgr at $30 \mathrm{kpc}$ along the line of sight. We have made a minor adjustment to the models to center them at precisely $\Lambda_{\odot}=0^{\circ}$ and $r=28$ or $30 \mathrm{kpc}$; although LM10a constrained their model to lie at $\Lambda_{\odot}=0^{\circ}$ and $r=28$, numerical limitations of the $N$-body method caused their model Sgr dSph to overshoot the correct position at the current epoch by $\sim 2^{\circ}$ (corresponding to $\sim 3 \mathrm{Myr}$ ) along its orbit. While this slight mismatch was unimportant for the analysis of LM10a (which focused on the properties of the Sgr tails at large angular separations from the dwarf), it is significant with respect to our present study of the Sgr core. We therefore manually wound the simulations back $\sim 3$ Myr to place the Sgr core at the fiducial location.

\subsection{The Breadth of the Sgr Stream}

The sky distribution of our Sgr detections (Figure 12 and the top panel of Figure 13) shows that the clusters and background features span the observed and modeled width of the stream. While we will address the distance measures in Section 4.3, the simple presence of Sgr debris is a useful constraint on Sgr's disruption. The detection of Sgr over such a large range in $B_{\odot}$ is consistent with the breadth of the stream depicted in M03 as well as the relatively high Sgr core mass used in the LM10a model. A smaller Sgr mass would result in narrower streams that would be inconsistent with the spatial distribution shown in the figures. For example, reducing the Sgr mass by a factor of two would leave the NGC 6652 and NGC 6637 Sgr features well outside the tidal stream (while also giving a stream velocity dispersion inconsistent with observations_-see Figure 4 of LM10a).

Expanding the range of Sgr pencil beams, especially across the face of the leading arm, would provide tighter constraints on the three-dimensional orientation of Sgr and the disruption models. A number of globular clusters are within the field depicted in Figure 13 (open squares in upper panel) ${ }^{14}$ and could potentially provide this additional constraint. Of the eleven clusters that could provide additional information, three have not been observed with HST/WFPC2 or HST/ACS. The remaining eight appear in Piotto et al. (2002), but the HST/WFPC2 data are not deep or precise enough to detect the faint Sgr stream. NGC 6522, NGC 6528, NGC 6544, NGC 6553, and NGC 6558 have been observed with the ACS but the data are not as deep as that of the ACS Treasury Program and lack the photometric precision to delineate the faint Sgr sequence. Published CMDs of NGC 6553 (Feltzing \& Gilmore 2000; Zoccali et al. 2001; Beaulieu et al. 2001) and NGC 6528 (Brown et al. 2005) do not show the stream clearly. Our deep precise HST imaging is the first to clearly discern these faint sequences.

Future deep observations of the above-named clusters-or any field within the Sgr stream-are recommended. Although the clusters marked in Figure 13 are farther away from the Sgr core - and would therefore have lower Sgr debris densities than even NGC 6652 - the mere presence or absence of the Sgr stream in these fields would help constrain the models of the

\footnotetext{
14 The clusters Djorg2, NGC 6522, NGC 6528, NGC 6540, NGC 6544, NGC 6553, NGC 6558, Terzan 12, NGC 6569, NGC 6626, and NGC 6638 would lie within the field of Figure 13.
} 


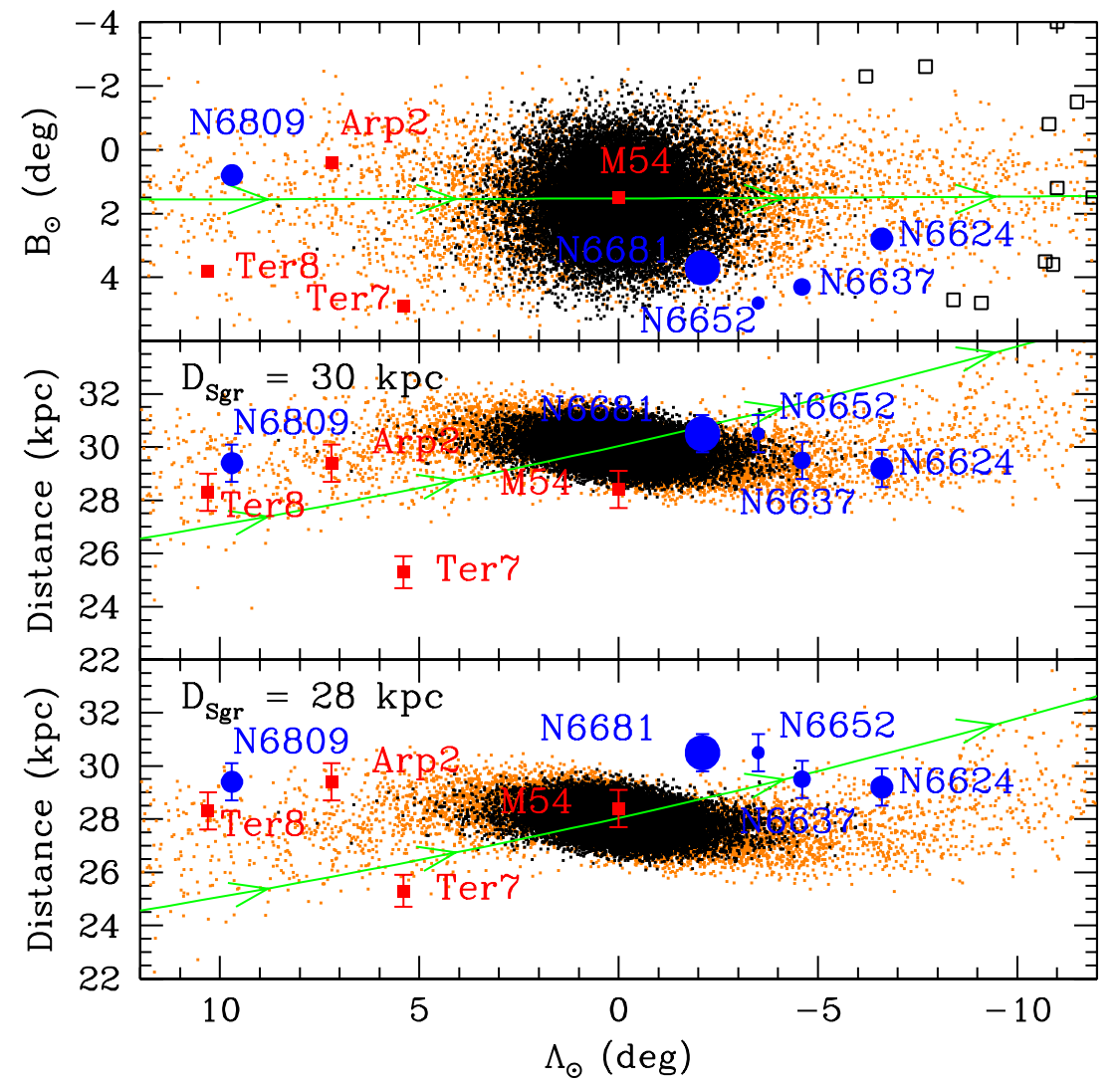

Figure 13. Distances and Sgr orbital latitude $B_{\odot}$ plotted against orbital longitude $\Lambda_{\odot}$ for the Sgr clusters (red squares) and Sgr CMD background features (blue circles) as well as globular clusters not included in the ACS Survey of Galactic Globular Clusters (open squares). The size of the Sgr main-sequence feature points is scaled to the number of Sgr member stars $\left(N_{\mathrm{Sgr}}\right)$ listed in Table 2. These are overlaid against the LM10a model of the Sgr tidal streams: black points are particles that are bound to the $\mathrm{dSph}$, while orange points are particles that have recently become unbound from the dwarf. The green line represents the orbital path of Sgr, with arrows representing its direction of motion. The middle panel shows the distance profile for a simulation in which the distance to Sgr has been set to $30 \mathrm{kpc}$. The bottom panel shows the distance profile for a simulation in which the distance to Sgr has been set to $28 \mathrm{kpc}$.

(A color version of this figure is available in the online journal.)

disrupting core by providing hard limits on the breadth of the stream in $B_{\odot}$. This is particularly true of NGC 6626/NGC 6638 and NGC 6558/NGC 6569, which occupy useful locations in the Sgr coordinate system $\left(\left(\Lambda_{\odot}, B_{\odot}\right) \sim(353,-2)\right.$ and $\sim(351,5)$, respectively).

The nearest clusters in our ACS program to the Sgr core, other than those in Table 2, are NGC 6656, 6717, and 6723 (marked as open circles in Figure 12). However, none of these clusters show any indication of Sgr debris. This is expected given the relatively high $B_{\odot}$ positions of the clusters $(-4.1,-6.1$, and +7.5 , respectively).

\subsection{The Density Distribution}

Figure 14 compares the density of Sgr main-sequence stars $\left(N_{\text {Sgr }}\right)$ measured in our five ACS fields against the power law+core density model fit to $\mathrm{M}$ giants from Table 1 of M03. The relative density level has been scaled to minimize the $\chi^{2}$ of the comparison without altering the core radius, ellipticity, or index of the M03 power-law model.

Four of the fields show consistent relative densities-within $1 \sigma-3 \sigma$ of the prediction. NGC 6652, however, represented in Figure 14 by an open square, is significantly $(7 \sigma)$ off from the prediction, despite its proximity to NGC 6637, which is at a similar radial distance and is the closest to the model prediction. Removing NGC 6652 from the fit would reduce the $\chi^{2}$ from 31 to 3.9 (dashed line in Figure 14).

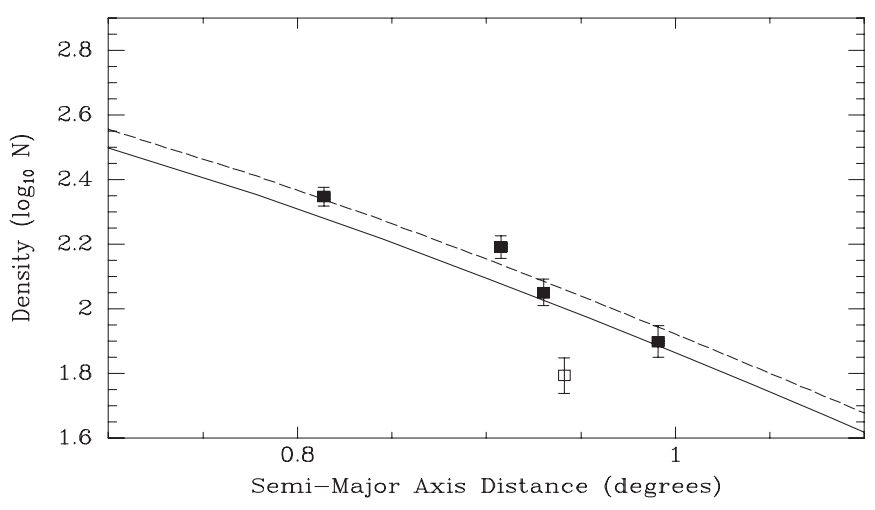

Figure 14. Comparison of the density of Sgr background debris to exponential profile described in Table 1 of M03. The profile has been arbitrarily scaled to match the mean density of the Sgr main-sequence stars. NGC 6652 (open square) has the lowest density of the data points. The lines show the best-fit density profile including NGC 6652 (solid line) and excluding it (dashed line).

We explored whether a change to the M03 model could bring the NGC 6652 data point back into line. Five data points are insufficient to constrain either a King or an exponential density model. However, we compared the data points to a series of "toy models" using the power law and core model from M03 with a variety of core radii, power-law indices, and ellipticities. The data were insensitive to the core radius and favored a slightly shallower power-law index than that of M03 (2.4-2.5, compared 
to M03's 2.6). We also found that the data favored a slightly higher ellipticity than M03's 0.62 of 0.67 (with NGC 6652) or 0.65 (without). However, even these changes only marginally improved the fit of the model, with and without NGC 6652, to $\chi^{2}$ values of 29 and 3.6, respectively. No simple exponential model could bring NGC 6652 into line with the other background features.

What could be the cause of the discrepancy in NGC 6652? Completeness is not an issue; the artificial star tests described in Paper V show that all five clusters have 90\%-95\% completeness levels to $F 814 W=23$. It is unlikely that any of our fields suffer from significant contamination from the foreground Galactic stars, despite their proximity to the midplane. CMDs for the non-Sgr detection fields of NGC 6656, 6717, and 6723, at comparable Galactic $b$ 's, show less than 10 stars in the CMD box from which $N_{\text {Sgr }}$ is calculated, which suggests a contamination level for NGC 6652 of less than 1/6 from all sources. The Besancon model (Robin et al. 2003) predicts only one to three Galactic halo stars in the CMD region from which we calculate $N_{\text {Sgr }}$. We could be suffering from photometric contamination from the foreground clusters in some of our fields. Some of the Sgr features-that of NGC 6681's CMD in particular-are close to the MS of the foreground cluster and it is possible that they suffer from some photometric contamination that inflates their densities slightly. However, it would take a significant (40\%) amount of hypothetical foreground contamination in the measured Sgr density in the other four clusters to make them consistent with NGC 6652.

If the density drop-off in NGC 6652 is real, it would indicate that while the density profiles fit in M03 provide a sound broad description of Sgr's density profile, the narrow field of ACS is more susceptible to small variations in density, such as might be produced by isophotal twisting or a transition from the bound core of Sgr to the unbound tidal stream (the contours of Ibata et al. 1995 hint at some irregularity in Sgr's inner structure). Far more extensive data will be needed to determine if the measured NGC 6652 field density of Sgr MS stars is simply a statistical anomaly or photometric error (albeit at the $7 \sigma$ level) or an indication of a much more complex inner structure in Sgr.

Comparing the Sgr stellar densities to the $N$-body model shows a rough agreement. NGC 6637 and 6652 are at large $B_{\odot}$ values while NGC 6809 and 6624 lie closer to the Sgr orbital plane. NGC 6681, which has the highest density, would be near the bound core of Sgr. The model does not explain the low density we measure for NGC 6652. However, models with a greater variety of Sgr core shapes (e.g., Łokas et al. 2010) may be better able to reproduce the observed sky densities.

\subsection{The Distance $-\Lambda_{\odot}-B_{\odot}$ Relations}

The $N$-body model of LM10a makes specific predictions about the relationship between sky position and line-of-sight distance for the disrupting Sgr dwarf. In the model, emerging debris in the trailing arm (positive $\Lambda_{\odot}$ values) is slightly more distant than emerging debris in the leading arm (negative $\Lambda_{\odot}$ ). This reflects the energy of the debris lost to the tails. The leading stream falls inside the orbital path and the angular momentum moves it ahead of Sgr along the orbit. The trailing stream falls outside the orbital path and the angular momentum moves it behind Sgr. Debris within $5^{\circ}$ of the Sgr core should follow this trend. According to the model, we should see an increase in distance with increasing $\Lambda_{\odot}$, albeit to a degree $(\sim 2 \mathrm{kpc})$ that would be close to the relative distance uncertainties in our study
(0.6-0.7 kpc assuming a relative distance modulus uncertainty of $0.05 \mathrm{mag})$.

At radii beyond $5^{\circ}$, the distance- $\Lambda_{\odot}$ trend reverses because of the shape of Sgr's orbit (the green line in Figure 13). The dSph is moving away from the Sun, so leading debris will be further away while trailing debris will be closer. According to the model, this orbital shape will result in a difference in line-of-sight distances of approximately $4 \mathrm{kpc}$ over the $\pm 12^{\circ}$ span of our survey. However, the combination of the two dynamical effects - mass loss mechanics and orbital path-should combine to make the distance to Sgr roughly constant over the $\Lambda_{\odot}$ interval covered by our data, with perhaps a slight overall trend of increasing distance with decreasing $\Lambda_{\odot}$.

Our points are close to the prediction of the model and the sense of the distance measures-either flat or slightly increasing distance with decreasing $\Lambda_{\odot}$-is similar to that of the unbound debris. However, with the exception of Terzan 7, the bottom panel of Figure 13 shows that our distance measures are consistently farther than predicted for a disrupting Sgr core at $28 \mathrm{kpc}$. Increasing the Sgr distance to $30 \mathrm{kpc}$, however (middle panel of Figure 13), brings the distance measures into remarkably improved agreement with the model. We explore the possibility of a larger Sgr distance further in Sections 4.4 and 4.5 .

\subsection{M54-Sgr Core or Chance Alignment?}

Bellazzini et al. (2008, hereafter B08) have argued, from measurements of velocity dispersion profiles, that M54 is not the core of Sgr but may have formed independently and plunged to the core of Sagittarius due to dynamical friction. Their dynamical simulations indicate that this process would take only a few gigayears. However, if M54 is liberated from its assumed status as the Sagittarius core, it need not necessarily be at the same distance along the line of site, as is assumed by B08.

If M54 were not precisely in the center of Sgr but were situated a kiloparsec or two in front of or in the back of the nucleus, then the Sgr distance in Paper IV would be accurate for M54 itself because that distance was measured from the dominant M54 metal-poor main sequence. However, the stellar populations within the parent $\mathrm{dSph}$ might be at a different distance. In Paper IV, we tied Sgr's distance to that of M54 because M54 dominates the main-sequence regions of the CMD and we could not discern a distance discrepancy between the two. The possibility of a distance difference between M54 and Sgr was not addressed.

Our new distance measures allow an independent constraint on the distance to Sgr in the absence of stars from the overlapping globular cluster. As noted above, the most striking aspect of Figure 13 is that two of the three Sgr canonical clusters and all five of the Sgr main-sequence features lie at the most distant edge of LM10a's theoretical Sgr stream for a distance of $28 \mathrm{kpc}$. Increasing the input model Sgr distance to $30 \mathrm{kpc}$ produces better agreement between the measured distances and the $N$-body model. Arp 2, Terzan 8, and all five background features would be comfortably within the Sgr stream at the longer distance (although Terzan 7 would be more discrepant). In this scenario, M54 would lie at the near edge of the dSph core, possibly still within the bound portions of the satellite but displaced from the center.

Could this apparent distance discrepancy be produced by something other than a genuine distance discrepancy between the Sgr core and M54? We consider five potential phenomena that could mimic a genuine discrepancy in distance between Sgr and M54. 
1. Observational error could produce an offset in the photometry that disguises itself as an apparent offset in distance. However, such a systematic error is unlikely. The chief advantage of the ACS survey is that the data are uniform, taken with the same instrument and filters over a short span of time and reduced and processed through an identical pipeline. For the distance difference to be a result of observational error, there would have to be a previously undetected change in the HST-ACS camera that affects all of the cluster fields except those of M54 and Terzan 7 (or, alternately, just those of M54 and Terzan 7).

2. We could be systematically overestimating the distance of the Sgr main-sequence features because of the small number of Sgr stars. Perhaps some of the brighter Sgr populations are too sparse to be clearly detected in the background features, causing us to shift the group of three isochrones, including the brighter ones, too faint in an effort to fit the data. However, NGC 6681, which has the strongest Sgr background signal, yields one of the most distant measures at $(m-M)_{0}=17.42$, a $3 \sigma$ discrepancy from the Paper IV Sgr distance (based on our estimate of 0.05 mag uncertainty in the relative distance).

3. Related to (2) is the possibility that we have overestimated the reddening, as discussed in Section 3.2. However, as noted in that section, all of our comparisons are relative. Moreover, NGC 6681, which has the strongest Sgr background signal, has an identical color shift to the central Sgr/M54 field but a large $(3 \sigma)$ magnitude shift. These discrepancies cannot be accounted for by errors in the assumed foreground reddening.

4. As noted above, our distance measures are sensitive to the assumed Sgr stellar populations used to constrain the distance to the background features. However, restoring the younger populations to our isochrones would result in a longer distance for the CMD background features. Removing the intermediate-age populations results in shorter distances for the Sgr background features. However, this results in a background Sgr population that is inconsistent with photometric measures of more dense areas of Sgr (see, e.g., Pryor et al. 2010) as well as spectroscopic surveys (P. Frinchaboy et al. 2011, in preparation) that definitively show an intermediate population consistent with our choice of isochrones. Moreover, the Sgr feature in NGC 6681 is very close to the Sgr core, perhaps within the bound remnant. It is unlikely that the background Sgr material in this field is much more metal-poor and/or older than the Sgr debris in the M54 field, given the abundances in the extended Sgr core and stream measured by Alard (2001), Chou et al. (2007, 2010), Giuffrida et al. (2010), and P. Frinchaboy et al. (2011, in preparation).

5. Finally, the notion that we can measure the distance of the Sgr core from measurements taken along the extended profile of Sgr assumes that we can use the LM10a model to compare the Sgr core distance to those measured along its tidal arms. It is remotely possible that an Sgr core at the same distance as M54 could have extended tidal arms that are at the distances measured in the other eight fields due to a different shape. However, this too is unlikely. It is difficult to configure Sgr's geometry so that both tidal arms are farther away than the Sgr core.

In the end, we conclude that the most likely explanation for the distance discrepancy between the Sgr debris and M54 is that M54 is, in fact, in front of the main body of Sgr. The

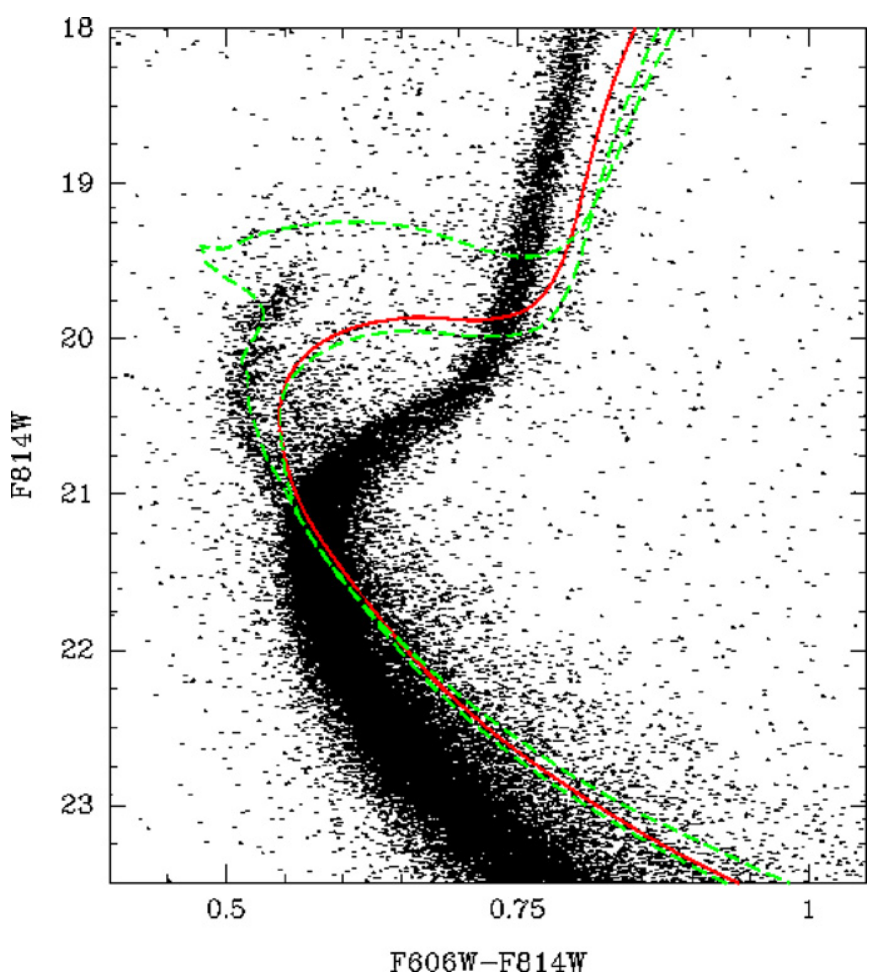

Figure 15. Effect of an increased Sgr distance modulus on the inferred stellar populations of M54/Sgr. The solid red line shows one of the SInt populations from Paper IV $([\mathrm{Fe} / \mathrm{H}]=-0.5,6 \mathrm{Gyr})$. The lower dashed green line shows a similar population $([\mathrm{Fe} / \mathrm{H}]=-0.4,5.5 \mathrm{Gyr})$ at the maximum distance modulus $\left((m-M)_{0}=17.39\right)$ implied by the bulge cluster Sgr CMD background features. The upper green dashed line shows the SYng population from Paper IV. The revision we have made to the isochrone color transformations for the younger isochrones (see the text in Section 4.4) cancels out the increase in distance modulus, resulting in a nearly identical fit $([\mathrm{Fe} / \mathrm{H}]=-0.2,2.8 \mathrm{Gyr})$.

(A color version of this figure is available in the online journal.)

mean distance modulus of the Sgr clusters is 17.22, shorter than the Paper IV distance of 17.27, mostly because of the short distance we measure for Terzan 7. Dropping Terzan 7 sets the mean distance modulus to 17.30. The CMD background features have a mean distance of 17.37 and a dispersion of 0.04 , indicating an Sgr distance modulus between 17.32 and 17.42 (assuming a $0.05 \mathrm{mag}$ relative uncertainty), corresponding to a linear distance of 29-30 kpc.

Increasing the Sgr distance, however, might change the assumed Sgr populations. The Sgr populations that we use to measure the distance of the background main-sequence features were constrained in Paper IV based on the assumption that they were at the same distance as M54. If the Sgr populations were more distant, however, this would alter the ages and metallicities of the Sgr populations described in Paper IV, which were based on the bluer MSTOs and RGBs of Sgr, rather than the M54-dominated MS. This could, in turn, change the distances measured for the background features from isochrone fitting.

However, our analysis indicates that these changes to the measured Sgr stellar populations would be minor. If we give Sgr a line-of-distance distance modulus of $(m-M)_{0}=17.39$, we could easily align the intermediate isochrones with the observed Sgr CMD features by decreasing their ages by $0.5-1$ Gyr and perhaps increasing their abundances by $0.1 \mathrm{dex}$ (see Figure 15). This would have little effect on the CMD background feature distances. Although the stellar population analysis of Paper IV was based on the MSTO and RGB, the distances in this 
paper were measured based solely on main-sequence fitting. Altering the metallicity of the intermediate-age populations by 0.1 dex would make the RGB/MSTO consistent with the greater distance modulus but not significantly change the mainsequence luminosity more than our uncertainties. The resultant distance measures would therefore be similar to those derived above. Increasing the distance to Sgr would not alter the Sgr MPP, since its properties were assumed, rather than fit, from the data owing to the M54 MPP dominating the main-sequence region of the CMD.

As for the younger populations characterized in Paper IV, our assessment of them would be little affected by an increase in distance. The youngest population (SVYng) is diffuse and its age/metallicity is more uncertain than the distance scale. The SYng population is better defined in the CMD. However, the increase in distance would almost exactly be cancelled by a change in isochrone calibration. Paper IV used semiempirical colors for the isochrones while this paper uses synthetic colors. While the different color transformations produce similar isochrones for the older populations, there is some disagreement as to the color and magnitudes of the younger populations. Given that the young populations are so far unconstrained by spectroscopic metallicities, their ages and metallicities are not well defined. Nevertheless, the SYng population described in Paper IV still accurately reproduces the overall shape and color of the SYng MSTO, as shown in Figure 15. Of course, neither SVYng nor SYng are seen in the Sgr main-sequence features, so they would not impact the distance measures in the bulge cluster fields.

Based on our present information, it is possible that M54 is not at the center of Sgr. Confirming or refuting this will require better constraints on Sgr's stellar populations outside of the central M54 field and/or more distance measures along the face of the dSph.

\subsection{The Status of the Classical Sgr Clusters}

One final problem related to using a longer distance for Sgr is the status of the globular cluster Terzan 7. Its distance of $25.4 \mathrm{kpc}$ would be consistent with the canonical Sgr distance used by previous investigations. However, it would be significantly $(6 \sigma$, assuming $\sigma_{m-M}=0.05$ ) different from the distance we measure for the other Sgr globular clusters $(28.5 \mathrm{kpc})$ and even more discrepant $(7 \sigma-9 \sigma)$ from the longer distance we argue to hold for the Sgr main body.

At first blush, it seems unlikely that Terzan 7 is only aligned with the Sgr dSph by chance. Terzan 7's age and metallicity are typical of the "young halo" objects traditionally associated with dSph galaxies. Terzan 7 and Pal 12 are the only globular clusters known to lack the $\mathrm{Na}-\mathrm{O}$ anticorrelation that is ubiquitous in Galactic globular clusters (Carretta et al. 2010a). Using previous distance measures, LM10b concluded that Terzan 7 was unlikely to be aligned by chance based on the cluster's location, radial velocity, and distance. However, we decided to re-examine the latter point in light of our new distance estimates.

To determine the status of Terzan 7, we re-ran the LM10a model and checked the status of all the Sgr member clusters for varying $\mathrm{Sgr}$ distances. If $\mathrm{Sgr}$ is at a distance of $28 \mathrm{kpc}$, Arp 2, Terzan 7, and Terzan 8, all can be matched with individual test particles in the Sgr simulation. In the simulation, the clusters have come unbound on the last pericenter $(0.1 \mathrm{Gyr}$ ago $)$ and will move much further $\left(70^{\circ}-110^{\circ}\right)$ downstream before the next pericenter. M54, of course, is in the center of Sgr in this simulation
Increasing the Sgr distance to $30 \mathrm{kpc}$ and placing M54 $2 \mathrm{kpc}$ in the foreground changes this picture somewhat. All four clusters match test particles within the Sgr debris, but Terzan 7 requires a much more generous search tolerance. Using the P3 statistic ${ }^{15}$ from LM10b, we find that Terzan 7's P3 value increases from 0.043 to 0.12 (compared to .001 for Terzan 8 and Arp 2). Given that $\sim 6$ such false positives expected at the level P3 $\sim 12 \%$, it seems possible that Terzan 7 could be a chance alignment. Given this now more troubling statistic, measuring an absolute proper motion for Terzan 7 is critical to affirming its status as a member of Sgr. If Terzan 7 is indeed a member, the distance discrepancy hints that Terzan 7 has a different dynamical history than the other classical Sgr member clusters.

As for M54, the simulation with the cluster in front of the dSph indicates that M54 should sink to the center of Sgr via dynamical friction in approximately $3 \mathrm{Gyr}$ if the dSph has a cuspy core consistent with the analysis of B08. This would seem to argue against the idea that M54 is 1-2 kpc in front of the dSph. However, if we assume a shallower density profile for Sgr, M54 may stall its descent near the core radius of $\sim 2 \mathrm{kpc}$ (M03). Given the similarity between this distance and our measured M54-Sgr distance $(2 \mathrm{kpc})$ this may already have happened, although it would seem a remarkable coincidence that M54 has stalled precisely along our line of sight to Sgr. ${ }^{16}$

\section{DISCUSSION AND CONCLUSIONS}

Our study of the Sagittarius system using ACS-WFC photometry has identified parts of the Sgr core and debris trail in the background of five unrelated bulge globular clusters. Assuming this background population to be consistent with the intermediate-age and old populations identified in Paper IV, we derive distances to these features.

The combination of five lines of sight to the Sgr main body as well as four Sgr member clusters allows nine precise and independent distance measures to the center of the Sgr system. The combined distances are roughly consistent with the Sgr distance measured in Paper IV for M54 $\left((m-M)_{0}=17.27\right.$; $d=28.4 \mathrm{kpc})$, which is slightly longer than the estimates based on the RGB tip (17.10; Monaco et al. 2004) and RR Lyrae stars (17.19; Layden \& Sarajedini 2000; Kunder \& Chaboyer 2009). However, the CMD background sequences are, in fact, slightly more distant than the Paper IV estimate. This may indicate that M54 - which is usually used as a proxy for measuring the distance to Sgr-lies in the foreground of the dwarf and that the true distance to Sgr is $\sim 2 \mathrm{kpc}$ larger than that derived in Paper IV. An increased Sgr distance would be more consistent with the distances we measure for both the background features and two of the other Sgr core globular clusters (Arp 2 and Terzan 8). If M54 were $2 \mathrm{kpc}$ closer along the line of sight, its distance would still be consistent with the "Sgr" distance measures made from RR Lyrae and RGB tip stars, measures that would be dominated by the M54 cluster. Dynamical models, both from B08 and LM10a, indicate that M54 would sink to the center of Sgr from dynamical friction in 2-3 Gyr. If it has not done so, this may indicate a shallower density profile for Sgr or that M54 has a different dynamical history than assumed.

Terzan 7 is several kiloparsecs closer to us than the main body of Sgr and the other core Sgr clusters. This raises the possibility

\footnotetext{
15 P3 is the probability that an artificial globular cluster inserted at random in the Galactic halo would by chance appear to match the Sgr stream as well as, or better than, the cluster in question.

16 In this scenario, M54's radial velocity, at this point in its orbit, would still be very close to that of the core, consistent with the measurement of B08.
} 


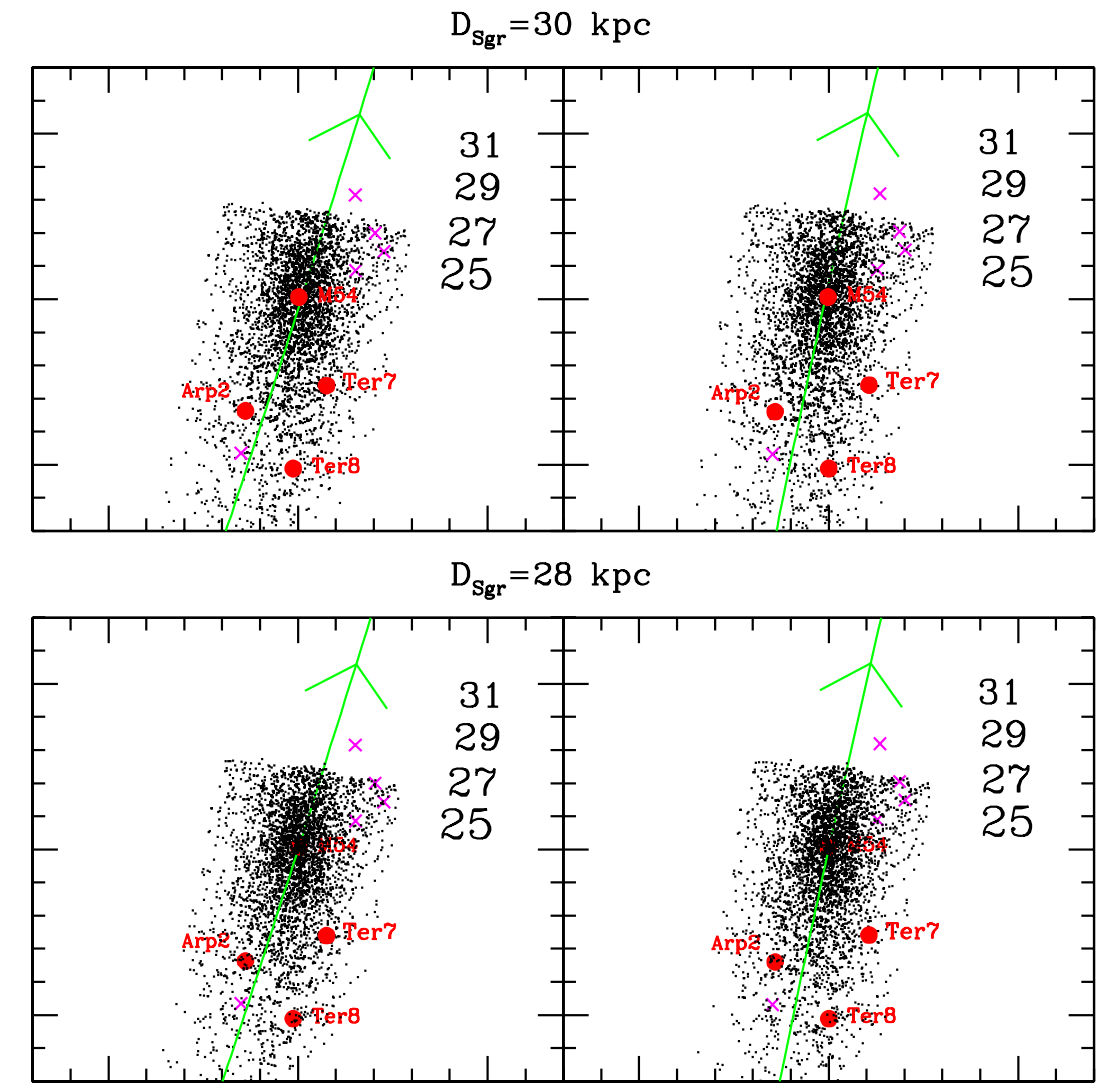

Figure 16. Sgr dwarf core and globular cluster system in a stereoscopic representation for a viewer at the location of the Sun with "eyes" $2.5 \mathrm{kpc}$ apart along the Galactic $Y$-axis (the direction of motion of the LSR). The view approximately has Galactic longitude along the horizontal axis (increasing from right to left) and Galactic latitude along the vertical axis (increasing from bottom to top), with a total field of view $\sim 30^{\circ} \times 30^{\circ}$. Blue crosses represent Sgr distances measured from the Sgr main-sequence features; red dots are the classical Sgr core globular clusters. Black points represent 2MASS M giants scaled to an Sgr core distance of the LM10a $N$-body model of $d_{\mathrm{Sgr}}=28 \mathrm{kpc}$ (lower panel) and $d_{\mathrm{Sgr}}=30 \mathrm{kpc}$ (upper panel). The M-giant distribution at the top of the image has been cut off because of contamination by disk M giants. The green line shows the orbit of Sgr, with the direction of motion indicated by the arrow. The depth gauge indicates the appearance of objects at distances of 25-31 kpc. These images are constructed in the cross-eyed stereoscopic format; the viewer can check that distances appear correctly by verifying that the $25 \mathrm{kpc}$ distance scale marker appears closer than the $31 \mathrm{kpc}$ marker. Both parallel view and cross-eyed view versions of this image are available at http://www.astro.virginia.edu/ srm4n/Sgr/stereo.html.

(A color version of this figure is available in the online journal.)

that Terzan 7 may not be part of Sgr or has a different dynamical history than that of the other globular clusters. Precise absolute proper motions for the cluster would determine which of these scenarios is more likely by tracing the orbit of Terzan 7.

We find that the stellar densities for the Sgr features are consistent with the elongated Sgr shape described in M03, which the exception of an unusually low density in the Sgr background feature of NGC 6652, which we cannot explain. The relative distance measures across the face of Sgr are also broadly consistent with expectations from current models of the Sgr tidal disruption (see, e.g., LM10a) as long as the longer distance scale (30 kpc) for the Sgr system is adopted. Our initial study indicates a substantial width to the emerging Sgr tidal tail, which confirms the higher Sgr mass used in the LM10a model.

To aid the reader in visualizing the relative three-dimensional orientation that our data imply for Sgr's inner regions, we have created a stereoscopic image of the Sgr system using our HST distance measures for the globular clusters and bulge cluster background CMD features, and the positions of the Two Micron All Sky Survey (2MASS) M giants (from Figure 4 of M03) with distances scaled to the LM10a simulation (Figure 16). The top panel shows a stereoscopic image with Sgr set at a distance of $30 \mathrm{kpc}$, and the bottom panel at $28 \mathrm{kpc}$. In these images, the green line represents the orbital path of the Sgr core with an arrow indicating its direction (i.e., toward the Galactic plane). The blue crosses show the relative distances measured for Sgr main-sequence debris stars in the background of the bulge clusters, the red dots are the classical Sgr clusters, and the points are 2MASS giants. Distances were assigned to the $\mathrm{M}$ giants schematically and guided by the $N$-body models so that, for each $\mathrm{M}$ giant, the distance corresponds to the distance of the $\mathrm{N}$-body model point that lies closest in projection. The stereoscopic image shows that M54 is either centered in the core of the Sgr dSph (bottom panel) or projected a few kpc in front of the core (top panel) depending on the chosen distance of the Sgr core. Our data clearly favor the longer distance-as evidenced in the stereoscopic image by the blue crosses lying among the $\mathrm{M}$ giants in depth perception for the $30 \mathrm{kpc}$ image, but behind the $\mathrm{M}$ giants in the $28 \mathrm{kpc}$ image. The stereoscopic image also makes evident that Terzan 7 is well in the foreground of the Sgr stars and other Sgr globular clusters no matter whether a $28 \mathrm{kpc}$ or $30 \mathrm{kpc}$ distance is adopted for the $\mathrm{Sgr}$ center. The $\mathrm{M}$ giants in the Sgr tail at the bottom of the image appear closer, following the general orientation of the Sgr orbit. ${ }^{17}$

\footnotetext{
${ }^{17}$ Both parallel view and cross-eyed view versions of this image are available at http://www.astro.virginia.edu/ srm $4 \mathrm{n} / \mathrm{Sgr} /$ stereo.html. To view the cross-eyed version presented here, the reader should slowly let their eyes cross until the images are doubled, then continue crossing their eyes until the middle images overlap, creating a three-dimensional perspective.
} 
Further studies of other fields within the Sgr core, perhaps using other bulge clusters as foreground reference points, would provide additional critical constraints on the emerging tidal debris. $N$-body simulations using a greater variety of Sgr core shapes and sizes would also allow a more robust comparison of the predicted properties of Sgr debris to our new measures. Finally, larger area surveys, such as the VVV survey of Minniti et al. (2010), will provide additional insight into the large- and small-scale structures of this intriguing dSph satellite galaxy of the Milky Way.

Support for this work (proposal number GO-10775) was provided by NASA through a grant from the Space Telescope Science Institute which is operated by the Association of Universities for Research in Astronomy, Incorporated, under NASA contract NAS5-26555. D.R.L. acknowledges support provided by NASA through Hubble Fellowship grant HF51244.01 awarded by the Space Telescope Science Institute. M.H.S. was supported at PSU by NASA contract NAS5-00136. S.R.M. was supported by NSF grant AST-0807945. This paper arose from discussion and work performed at the Aspen Center for Physics, which is supported by NSF and NASA funding.

\section{REFERENCES}

Alard, C. 2001, A\&A, 377, 389

Anderson, J., Sarajedini, A., Bedin, L. R., et al. 2008, AJ, 135, 2055 (Paper V) Beaulieu, S. F., Gilmore, G., Elson, R. A. W., et al. 2001, AJ, 121, 2618 Bedin, L. R., Cassisi, S., Castelli, F., et al. 2005, MNRAS, 357, 1038 Bellazzini, M., Correnti, M., Ferraro, F. R., Monaco, L., \& Montegriffo, P. 2006, A\&A, 446, L1

Bellazzini, M., Ferraro, F. R., \& Ibata, R. 2002, AJ, 124, 915

Bellazzini, M., Ferraro, F. R., \& Ibata, R. 2003a, AJ, 125, 188

Bellazzini, M., Ibata, R., Ferraro, F. R., \& Testa, V. 2003b, A\&A, 405, 577

Bellazzini, M., Ibata, R. A., Chapman, S. C., et al. 2008, AJ, 136, 1147 (B08)

Belokurov, V., Zucker, D. B., Evans, N. W., et al. 2006, ApJ, 642, L137

Bohlin, R. C. 2007, Instrument Science Report ACS 2007-06, 6, 25 pp

Brown, T. M., Ferguson, H. C., Smith, E., et al. 2005, AJ, 130, 1693

Carraro, G. 2009, AJ, 137, 3809

Carraro, G., Zinn, R., \& Moni Bidin, C. 2007, A\&A, 466, 181

Carretta, E., Bragaglia, A., Gratton, R. G., et al. 2010a, A\&A, 516, A55

Carretta, E., Bragaglia, A., Gratton, R. G., et al. 2010b, ApJ, 714, L7

Chou, M.-Y., Cunha, K., Majewski, S. R., et al. 2010, ApJ, 708, 1290

Chou, M.-Y., Majewski, S. R., Cunha, K., et al. 2007, ApJ, 670, 346

Cohen, J. G. 2004, AJ, 127, 1545

Correnti, M., Bellazzini, M., Ibata, R. A., Ferraro, F. R., \& Varghese, A. 2010, ApJ, 721, 329

Da Costa, G. S., \& Armandroff, T. E. 1995, AJ, 109, 2533

Dinescu, D. I., Majewski, S. R., Girard, T. M., \& Cudworth, K. M. 2000, AJ, 120, 1892

Dotter, A., Chaboyer, B., Jevremović, D., et al. 2007, AJ, 134, 376

Dotter, A., Sarajedini, A., Anderson, J., et al. 2010, ApJ, 708, 698 (Paper IX)
Fellhauer, M., Belokurov, V., Evans, N. W., et al. 2006, ApJ, 651, 167

Feltzing, S., \& Gilmore, G. 2000, A\&A, 355, 949

Gilliland, R. 2004, ACS/ISR 2004-01, CCD Gains, Full Well Depths, and Linearity up to and Beyond Saturation

Giuffrida, G., Sbordone, L., Zaggia, S., et al. 2010, A\&A, 513, A62

Goldsbury, R., Richer, H. B., Anderson, J., et al. 2010, AJ, 140, 1830

Harris, W. E. 1996, AJ, 112, 1487

Helmi, A. 2004, MNRAS, 351, 643

Ibata, R., Lewis, G. F., Irwin, M., Totten, E., \& Quinn, T. 2001, ApJ, 551, 294

Ibata, R. A., Gilmore, G., \& Irwin, M. J. 1994, Nature, 370, 194

Ibata, R. A., Gilmore, G., \& Irwin, M. J. 1995, MNRAS, 277, 781

Johnston, K. V., Law, D. R., \& Majewski, S. R. 2005, ApJ, 619, 800

Kunder, A., \& Chaboyer, B. 2009, AJ, 137, 4478

Law, D. R., Johnston, K. V., \& Majewski, S. R. 2005, ApJ, 619, 807

Law, D. R., \& Majewski, S. R. 2010a, ApJ, 714, 229 (LM10a)

Law, D. R., \& Majewski, S. R. 2010b, ApJ, 718, 1128 (LM10b)

Law, D. R., Majewski, S. R., \& Johnston, K. V. 2009, ApJ, 703, L67

Layden, A. C., \& Sarajedini, A. 2000, AJ, 119, 1760

Łokas, E. L., Kazantzidis, S., Majewski, S. R., et al. 2010, ApJ, 725, 1516

Majewski, S. R., Skrutskie, M. F., Weinberg, M. D., \& Ostheimer, J. C. 2003, ApJ, 599, 1082 (M03)

Marín-Franch, A., Aparicio, A., Piotto, G., et al. 2009, ApJ, 694, 1498 (Paper VII)

Marshall, D. J., Robin, A. C., Reylé, C., Schultheis, M., \& Picaud, S. 2006, A\&A, 453,635

Martínez-Delgado, D., Peñarrubia, J., Jurić, M., Alfaro, E. J., \& Ivezić, Z 2007, ApJ, 660, 1264

Mateo, M., Mirabal, N., Udalski, A., et al. 1996, ApJ, 458, L13

Milone, A. P., Bedin, L. R., Piotto, G., et al. 2008, ApJ, 673, 241

Minniti, D., Lucas, P. W., Emerson, J. P., et al. 2010, New Astron., 15, 433

Monaco, L., Bellazzini, M., Ferraro, F. R., \& Pancino, E. 2004, MNRAS, 353, 874

Mottini, M., Wallerstein, G., \& McWilliam, A. 2008, AJ, 136, 614

Niederste-Ostholt, M., Belokurov, V., Evans, N. W., \& Peñarrubia, J. 2010, ApJ, 712,516

Palma, C., Majewski, S. R., \& Johnston, K. V. 2002, ApJ, 564, 736

Paust, N. E. Q., Aparicio, A., Piotto, G., et al. 2009, AJ, 137, 246

Paust, N. E. Q., Reid, I. N., Piotto, G., et al. 2010, AJ, 139, 476

Peñarrubia, J., Belokurov, V., Evans, N. W., et al. 2010, MNRAS, 408, L26

Peñarrubia, J., Zucker, D. B., Irwin, M. J., et al. 2011, ApJ, 727, L2

Piotto, G., King, I. R., Djorgovski, S. G., et al. 2002, A\&A, 391, 945

Pryor, C., Piatek, S., \& Olszewski, E. W. 2010, AJ, 139, 839

Riess, A., \& Mack, J. 2004, ACS/ISR 2004-06, Time Dependence of ACS CTE Corrections for Photometry and Future Predictions

Robin, A. C., Reylé, C., Derrière, S., \& Picaud, S. 2003, A\&A, 409, 523

Sarajedini, A., Bedin, L. R., Chaboyer, B., et al. 2007, AJ, 133, 1658 (Paper I)

Sbordone, L., Bonifacio, P., Marconi, G., Buonanno, R., \& Zaggia, S. 2005, A\&A, 437, 905

Schlegel, D. J., Finkbeiner, D. P., \& Davis, M. 1998, ApJ, 500, 525

Siegel, M. H., Dotter, A., Majewski, S. R., et al. 2007, ApJ, 667, L57 (Paper IV)

Sirianni, M., Jee, M. J., Benítez, N., et al. 2005, PASP, 117, 1049

Sollima, A., Cacciari, C., Bellazzini, M., \& Colucci, S. 2010, MNRAS, 406, 329

Yanny, B., Newberg, H. J., Johnson, J. A., et al. 2009, ApJ, 700, 1282

Zoccali, M., Renzini, A., Ortolani, S., Bica, E., \& Barbuy, B. 2001, AJ, 121, 2638 